\title{
The intron-enriched HERV-K(HML-10) family suppresses apoptosis, an indicator of malignant transformation
}

\author{
Felix Broecker ${ }^{1,2,3^{*}}$, Roger Horton ${ }^{1}$, Jochen Heinrich², Alexandra Franz ${ }^{1,4}$, Michal-Ruth Schweiger ${ }^{1,5}$, \\ Hans Lehrach ${ }^{1,6}$ and Karin Moelling ${ }^{1,2}$
}

\begin{abstract}
Background: Human endogenous retroviruses (HERVs) constitute $8 \%$ of the human genome and contribute substantially to the transcriptome. HERVs have been shown to generate RNAs that modulate host gene expression. However, experimental evidence for an impact of these regulatory transcripts on the cellular phenotype has been lacking.

Results: We characterized the previously little described HERV-K(HML-10) endogenous retrovirus family on a genome-wide scale. HML-10 invaded the ancestral genome of Old World monkeys about 35 Million years ago and is enriched within introns of human genes when compared to other HERV families. We show that long terminal repeats (LTRs) of HML-10 exhibit variable promoter activity in human cancer cell lines. One identified HML-10 LTR-primed RNA was in opposite orientation to the pro-apoptotic Death-associated protein 3 (DAP3). In HeLa cells, experimental inactivation of HML-10 LTR-primed transcripts induced DAP3 expression levels, which led to apoptosis.

Conclusions: Its enrichment within introns suggests that HML-10 may have been evolutionary co-opted for gene regulation more than other HERV families. We demonstrated such a regulatory activity for an HML-10 RNA that suppressed DAP3-mediated apoptosis in HeLa cells. Since HML-10 RNA appears to be upregulated in various tumor cell lines and primary tumor samples, it may contribute to evasion of apoptosis in malignant cells. However, the overall weak expression of HML-10 transcripts described here raises the question whether our result described for HeLa represent a rare event in cancer. A possible function in other cells or tissues requires further investigation.
\end{abstract}

Keywords: Apoptosis, Cancer, DAP3, Death-associated protein 3, Endogenous retrovirus, Gene regulation, Genome evolution, HERV, HERV-K(HML-10)

\section{Background}

About half of the human genome is composed of transposable elements (TEs) [1], and recent evidence suggests even a fraction of up to two thirds [2]. The most abundant TEs in the human genome are retroelements (REs) that amplify via a 'copy-and-paste' mechanism involving reverse transcription of an RNA intermediate $[1,3]$.

\footnotetext{
*Correspondence: felixbroecker@gmx.net

'Max Planck Institute for molecular Genetics, Ihnestr. 63-73, 14195 Berlin, Germany

${ }^{2}$ Institute of Medical Microbiology, University of Zurich, Gloriastr. 32, 8006

Zurich, Switzerland

Full list of author information is available at the end of the article
}

One class of REs, HERVs, comprises remnants of ancient retroviral germ line cell infections that became evolutionary fixed in the genome. About 450,000 HERV elements constitute $8 \%$ of the human genome and are classified into about 30 families $[1,4]$. HERVs are structurally similar to proviruses of present-day retroviruses where the gag, pol and env genes are flanked by two long terminal repeats (LTRs) that act as promoters [4]. HERVs and other REs have been shown to influence gene regulation by providing regulatory elements such as enhancers, promoters, splice- and polyadenylation sites, for various host genes [3]. REs of all classes often contain functional promoters and consequently 
contribute to a large fraction of the human transcriptome [5]. Numerous REs are located within introns of host genes and might be involved in antisense gene regulation in cis [1]. The potential significance of RE-mediated cis-antisense gene regulation is suggested by the genome-wide presence of about 48,000 transcription start sites (TSSs) within HERVs and other REs that are in reverse orientation to overlapping host genes [6].

Promoter activity, a prerequisite for REs to exert antisense-mediated gene regulation, has been shown for representative LTRs of HERV-E [7], HERV-W [8], HERV-H [9-12], HERV-L [9], HERV-I [13] and HERV-K(HML-2), HML standing for human mouse mammary tumor viruslike [14-17]. The latter HERV family, HML-2 in the following, is the phylogenetically most recent and most active one in the human genome $[3,4]$, with about $50 \%$ of LTRs being transcriptionally active [15]. Antisense gene regulation in cis has been shown for HML-2 LTRs located within introns of the SLCAA8 (a sodium bicarbonate co-transporter) and IFT172 (intraflagellar transport protein 172) genes [14]. In addition, the PLA2G4A gene that encodes a phospholipase with a possible implication in tumorigenesis is negatively regulated by a HERV-E LTR-primed transcript [7]. These three individual cases are presently the only experimentally verified examples of the influence of LTR-primed transcripts on gene regulation.

A HERV family phylogenetically related to HML-2 is HERV-K(HML-10), HML-10 in the following [4]. The prototypical HML-10 provirus located within an intron of the long variant of the Complement Component 4 (C4) gene has been shown to possess promoter activity in its $3^{\prime}$ LTR $[18,19]$. Since this provirus remains the only one studied in detail to date, we here characterized the HML-10 family in more detail. We found that HML-10 invaded the ancestral genome of the Old World monkey (OWM) lineage about 35 Mya. A survey of the human genome revealed that HML-10 sequences were significantly enriched within host gene introns, indicating their evolutionary recruitment for gene regulatory functions. Three intron-located HML-10 proviruses exerted LTR promoter activity in the human HEK293T and HepG2 tumor cell lines in vitro. Transcriptional orientation and strength varied substantially between the cell lines and promoter activity was suppressed by interferon-gamma (IFNY). One of the proviral LTRs showed transcriptional activity in opposite orientation to the encompassing pro-apoptotic $D A P 3$ gene that encodes a signaling protein of the Death Receptor (DR) pathway [20, 21]. We provide evidence that HML-10 LTR-primed transcripts negatively regulate DAP3 expression in HeLa cells, as their inactivation by antisense oligonucleotides (ASOs) led to a 10-fold increase in DAP3 mRNA levels and efficiently promoted apoptosis. Our findings support the functional relevance of LTR-primed cisregulatory transcripts for human gene regulation and the cellular phenotype and function.

\section{Results}

HML-10 elements are 35 million years old and enriched within human genes

To identify potential priming of cis-acting regulatory transcripts by HERVs, we mined the GRCh38/hg38 human genome assembly [1] for sequences of the previously little described HML-10 family. The prototype member of HML-10 is an intron-located provirus in the long form of the $C 4$ gene that exhibits LTR promoter activity in vitro $[18,19]$. Expression of this provirus has been detected via microarray before, for instance, in brain, breast, kidney and skin tissue, blood cells as well as various human cancer cell lines [22-27].

The provirus inside of the $C 4$ gene is currently the only HML-10 sequence described in the literature $[18,19]$. With a size of about 6400 basepairs (bp) it contains the retroviral gag, pol and env genes, an A/T-rich stretch of unknown function between $p o l$ and env and two flanking LTRs [18] (Fig. 1a). Most HERV elements found in the human genome today have undergone homologous recombination between their two proviral LTRs, leaving behind solitary LTRs $[1,3,4]$ that in this case have a size of about $550 \mathrm{bp}$. We identified seventy HML-10 elements within the human genome (Table 1). Of these, seven are proviruses with the structure 5'LTR-gag-pol-A/T-rich-env-3'LTR (with element no. 58 lacking the 5'LTR) and 63 are solitary LTRs. Some of the elements are truncated at either end or harbor other REs, mostly Alus. HERV sequences can be amplified by chromosomal duplication events following integration [4]. To reveal whether the identified HML-10 elements represent independent integration events, we determined their target site duplications (TSDs). The TSDs were expected to differ between independently acquired HML-10 elements. It has been shown previously that the provirus in the $C 4$ gene (element no. 22) created a 6 bp TSD [18]. Confirming these findings, we could identify TSDs of 5 or 6 bp for most (59 of 70) HML-10 elements (Table 1). All identified TSDs had a unique sequence, whereby the two copies of element no. 22 showed an identical 6 bp TSD with the expected sequence [18]. Alignment of the flanking regions of each HML-10 element $( \pm 1000 \mathrm{bp})$ revealed no sequence homology except for the two proviruses of element no. 22 as well as between elements nos. 27 and 45 (Additional file 1: Figure S1). Thus, one of the latter two has arisen through chromosomal duplication and the other 69 HML-10 elements listed in Table 1 are likely the result of independent retroviral integration events.

To reveal the evolutionary history of HML-10, we first searched for HML-10 sequences in genomes of different mammalian species. HML-10 was identified in all investigated genomes of the OWM lineage, but was absent in the genomes of New World monkeys (NWMs) and the more distantly related species mouse lemur, bushbaby and mouse 

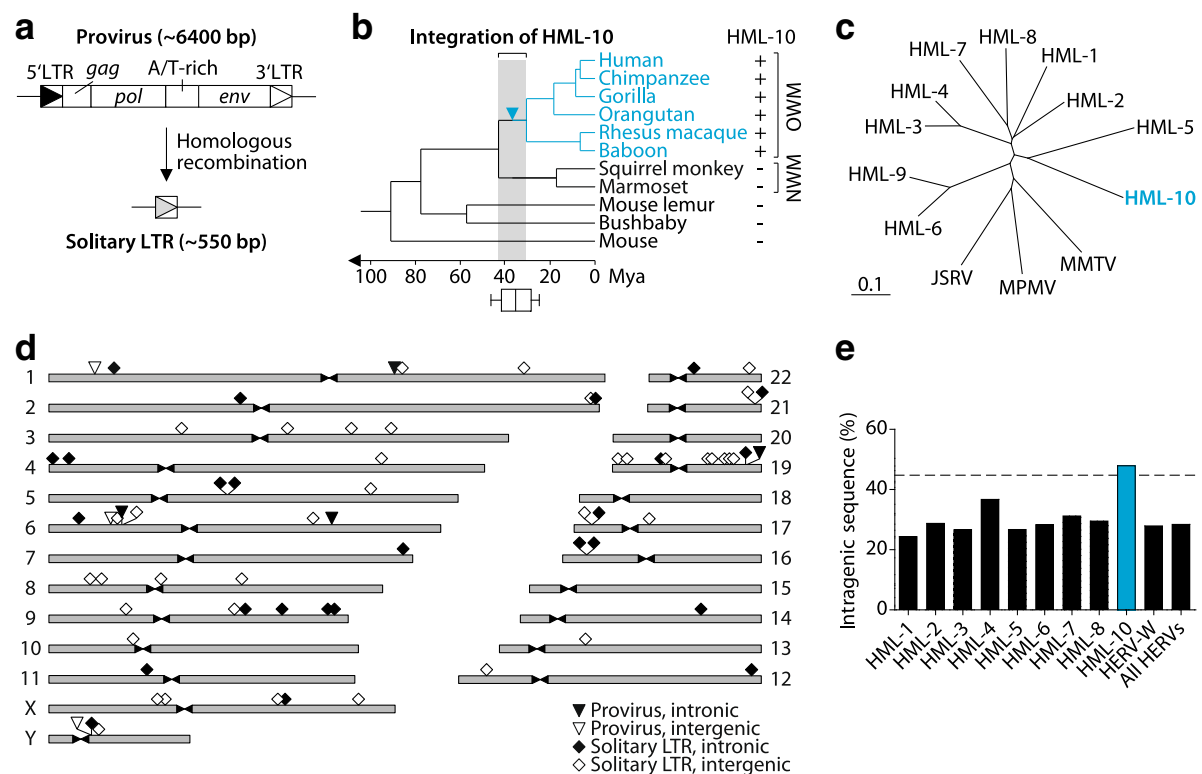

e

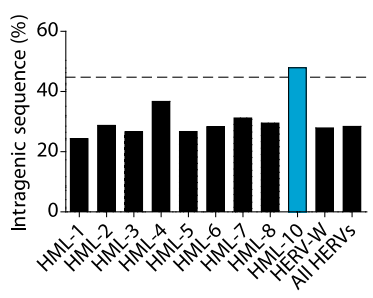

Fig. 1 Characteristics of the HML-10 endogenous retrovirus family. a Structure of HML-10 proviruses [18]. b Estimation of the evolutionary age of HML-10 with divergence times as reported before [78]. The box-and-whiskers plot shows age estimation by sequence comparison of LTRs from six complete proviruses (elements nos. 1, 3, 20, 22, 25 and 68 in Table 1) in the human genome [28]. The arrowhead indicates the integration events in the OWM lineage. c Neighbor-joining tree of Pol protein sequences of different endogenous and exogenous betaretroviruses [18, 72]. The horizontal bar represents 0.1 substitutions per amino acid position. $\mathbf{d}$ Chromosomal distribution of HML-10 elements in the human genome. Details can be found in Table 1. e Comparison of genomic fractions of intragenic elements (located within the boundaries of RefSeq [33] genes) between HML-10 and other HERV families in the human genome. All observed distributions differed significantly from the expected value for random integration that is shown as dotted horizontal line, with $P$-values $\leq 0.01$ inferred by chi-square tests

(Fig. 1b). The OWM genomes contained between 80 and 96 HML-10 sequences (Additional file 2: Table S1). Of note, about 600 sequences annotated as HML-10 by RepeatMasker were found in the investigated NWM genomes that however shared little sequence homology with the ones found in OWMs. Thus, the annotated HML-10 elements in OWM and NWM genomes likely represent two distinct HERV families.

The evolutionary age of HML-10 was estimated by calculating the nucleotide sequence divergence between both LTRs of each of the six complete proviruses (Table 1), applying a mutation rate of 2.28 substitutions per site and year $\times 10^{-9}$ as described [28]. This analysis yielded an evolutionary age of $35.3 \pm 7.8$ million years (mean \pm SD, see box-and-whiskers plot in Fig. 1b). Phylogenetic neighbor-joining analysis of 68 complete human HML-10 LTRs, including both LTRs of each of six complete proviruses, revealed a near-monophyletic tree (Fig. 2), indicating a single integration period. Therefore, the infectious progenitor of HML-10 likely invaded the ancestral genomes of OWM during a brief period around $35 \mathrm{Mya}$ (Fig. 1b). The same age has been attributed before to other endogenous human betaretrovirus families, including HML-2 [4], HML-3 [29], HML-4 [30] and HML-6 [31]. In contrast, the HML-5 infectious progenitor was active about 55 Mya [32] and HML-2 has remained active after the divergence of humans and chimpanzees about six Mya [4]. Neighbor-joining analysis of pol sequences of various endogenous and exogenous betaretroviruses showed that HML-10 is closely related to HML-1 through HML-9 HERVs and the extant exogenous retroviruses JSRV (Jaagsiekte sheep retrovirus), MPMV (Mason-Pfizer monkey virus) and MMTV (mouse mammary tumor virus) (Fig. 1c).

HML-10 elements are non-randomly distributed among human chromosomes (Fig. 1d). Most notably, the relatively small chromosome 19 harbored the highest number of elements (11 of 70$)$. This was a first indication that HML-10 sequences were preferentially located near host genes, since chromosome 19 is the most gene-dense one [1]. Of the 70 HML-10 elements, 29 (41.4\%) were found within introns of human genes (as annotated by RefSeq [33]), and 16 of the remaining 41 intergenic elements were located in proximity $( \pm 10,000 \mathrm{bp})$ to at least one RefSeq gene (Table 1$)$. The relatively frequent location of HML-10 in the vicinity of host genes is a feature that is not shared with other HERV families studied in this regard. Namely, only $28 \%$ of all HERV-W elements are located within introns of genes [8] and HML-2 was found to be enriched outside genes, although de novo infection and integration of a resurrected HML-2 retrovirus favored actively transcribed regions [34], 
Table $1 \mathrm{HML}-10$ elements in the human genome

\begin{tabular}{|c|c|c|c|c|c|c|c|c|}
\hline No. & Chr.band & Coordinates (strand) & Structure & TSD & Integrated REs & Position & $\begin{array}{l}\text { RefSeq genes } \\
\text { (rel. orientation) }\end{array}$ & $\begin{array}{l}\text { RefSeq genes } \\
\pm 10,000 \text { bp }\end{array}$ \\
\hline 1 & $1 p 36.13$ & chr1:19926886-19932710 (-) & Provirus & WWAAAT & - & Intergenic & - & PLA2G2E \\
\hline 2 & $1 \mathrm{p} 35.3$ & chr1:29156730-29157306 (-) & LTR & GTTAC & - & Intronic & SFRS4 (s) & - \\
\hline 3 & $1 q 22$ & chr1:155691832-155699521 (-) & Provirus & ATTAAG & AluSp; MER11B & Intronic & DAP3 (as) & YY1AP1 \\
\hline 4 & $1 \mathrm{q} 23.1$ & chr1:158534566-158535143(+) & LTR & GTCCAA & - & Intergenic & - & - \\
\hline 5 & $1 \mathrm{q} 32.3$ & chr1:213045116-213046160 (-) & $\operatorname{LTR}^{\mathrm{a}}$ & TAGTGG & - & Intergenic & - & RPS6KC1 \\
\hline 6 & $2 \mathrm{p} 11.2$ & chr2:86245199-86245755 (+) & LTR & TATAC & - & Intronic & REEP1 (as) & - \\
\hline 7 & $2 q 37.3$ & chr2:238659222-238659778 (+) & LTR & not found & - & Intergenic & - & - \\
\hline 8 & $2 q 37.3$ & chr2:240556198-240556747 (+) & LTR & not found & - & Intronic & ANKMY1 (as) & DUSP28 \\
\hline 9 & $3 p 14.2$ & chr3:58724279-58724839 (+) & LTR & CAGCAG & - & Intergenic & - & - \\
\hline 10 & $3 q 12.2$ & chr3:101089113-101089658 (+) & LTR & AGGCAC & - & Intergenic & - & - \\
\hline 11 & $3 q 21.3$ & chr3:128828801-128835173 (+) & LTR & TGCAT & $\begin{array}{l}\text { AluY; LTR7B; } \\
\text { HERVH }\end{array}$ & Intergenic & - & - \\
\hline 12 & $3 q 24$ & chr3:146283898-146284161 (-) & $\operatorname{LTR}^{b}$ & not found & - & Intergenic & - & - \\
\hline 13 & $4 \mathrm{p} 16.3$ & chr4:1709255-1710065 (+) & LTR & ATGGGG & AluY & Intronic & SLBP (as) & TMEM129 \\
\hline 14 & $4 p 16.1$ & chr4:8441728-8442282 (-) & LTR & YYTTTA & - & Intronic & TRMT (as) & ACOX3 \\
\hline 15 & $4 q 31.21$ & chr4:143777776-143778330(+) & LTR & TCARCC & - & Intergenic & - & - \\
\hline 16 & $5 q 14.1$ & chr5:78245022-78245575 (-) & LTR & TCYWCA & - & Intronic & $A P 3 B 1(\mathrm{~s})$ & - \\
\hline 17 & $5 q 14.1$ & chr5:78248004-78248093 (-) & $\operatorname{LTR}^{\mathrm{b}}$ & not found & - & Intronic & AP3B1 (s) & - \\
\hline 18 & $5 q 31.3$ & chr5:142088759-142089306 (-) & LTR & TTGGTG & - & Intergenic & - & - \\
\hline 19 & $6 \mathrm{p} 24.1$ & chr6:12989575-12990131 (-) & LTR & GAAAAC & - & Intronic & PHACTR1 (as) & - \\
\hline 20 & $6 \mathrm{p} 22.1$ & chr6:27187520-27196279 (+) & Provirus & AAGATM & $\begin{array}{l}\text { 3× AluY; } \\
\text { AluYc; LTR13A }\end{array}$ & Intergenic & - & - \\
\hline 21 & $6 \mathrm{p} 22.1$ & chr6:28607908-28608446 (+) & LTR & CATGTT & - & Intergenic & - & - \\
\hline 22 & $6 p 21.33$ & $\begin{array}{l}\text { chr6:31984691-31991052 (-) } \\
\text { chr6:32017429-32023790 (-) }\end{array}$ & Provirus & $\begin{array}{l}\text { TGTCTG } \\
\text { TGTCTG }\end{array}$ & - & Intronic & $C 4 A / C 4 B$ (as) & $\begin{array}{l}\text { CYP21A1P; } \\
\text { STK19; TNXB }\end{array}$ \\
\hline 23 & $6 p 21.32$ & chr6:32512829-32513385 (+) & LTR & GGGGRG & - & Intergenic & - & HLA-DRB5 \\
\hline 24 & $6 q 21$ & chr6:114011436-114018365 (+) & LTR & СССТАТ & LTR7B; HERVH & Intergenic & - & - \\
\hline 25 & $6 q 22.31$ & chr6:122504844-122512093 (-) & Provirus & GGACAT & $3 \times$ AluY & Intronic & PKIB (as) & - \\
\hline 26 & $7 q 36.2$ & chr7:154936774-154937317 (-) & LTR & ACTCCA & - & Intronic & PAXIP1 (as) & PAXIP1-AS2 \\
\hline 27 & $8 p 22$ & chr8:17915846-17916036 (-) & $\operatorname{LTR}^{\mathrm{b}}$ & CCCMTA & - & Intergenic & - & PCM1 \\
\hline 28 & $8 p 21.3$ & chr8:22985089-22985644 (-) & LTR & CCTCYY & - & Intergenic & - & RHOBTB2 \\
\hline 29 & $8 q 11.1$ & chr8:46533684-46534254 (+) & LTR & CATTTC & - & Intergenic & - & - \\
\hline 30 & $8 q 21.13$ & chr8:82206225-82206776 (+) & LTR & CASCCK & - & Intergenic & - & - \\
\hline 31 & $9 p 13.3$ & chr9:34539821-34540365 (-) & LTR & GGCATG & - & Intergenic & - & - \\
\hline 32 & $9 q 22.1$ & chr9:87984145-87984721 (-) & LTR & TATGGC & - & Intergenic & - & CDK20 \\
\hline 33 & $9 q 22.31$ & chr9:92523403-92523958 (-) & LTR & WATTGT & - & Intronic & CENPP (as); ECM2 (s) & - \\
\hline 34 & $9 q 31.3$ & chr9:109072071-109072632 (+) & LTR & CMAAAG & - & Intronic & TMEM245 (as) & - \\
\hline 35 & $9 q 34.11$ & chr9:129101280-129101834 (+) & LTR & GGGGAA & - & Intronic & CRAT (as) & PPP2R4 \\
\hline 36 & $9 q 34.13$ & chr9:132289026-132289571 (+) & LTR & CTCTYA & - & Intronic & SETX (as) & - \\
\hline 37 & 10p11.21 & chr10:37685164-37685717 (+) & LTR & GAATC & - & Intergenic & - & - \\
\hline 38 & $11 \mathrm{p} 11.2$ & chr11:43747422-43747974 (+) & LTR & GTTCTG & - & Intronic & HSD17B12 (s) & - \\
\hline 39 & 12p13.1 & chr12:12810288-12810845 (-) & LTR & ATCTA & - & Intergenic & - & $D D \times 47$ \\
\hline 40 & $12 q 24.33$ & chr12:132949015-132949570 (+) & LTR & GTATC & - & Intronic & ZNF605 (as) & - \\
\hline 41 & $13 q 13.3$ & chr13:37090225-37090778 (+) & LTR & CCTGTT & - & Intergenic & - & - \\
\hline
\end{tabular}


Table 1 HML-10 elements in the human genome (Continued)

\begin{tabular}{|c|c|c|c|c|c|c|c|c|}
\hline 42 & $14 q 31.1$ & chr14:79706443-79707024 (+) & LTR & TTGGTC & - & Intronic & NRXN3 (s) & - \\
\hline 43 & $16 p 13.13$ & chr16:10785748-10785943 (+) & $\mathrm{LTR}^{\mathrm{b}}$ & not found & - & Intronic & FAM18A (as) & - \\
\hline 44 & $16 p 13.13$ & chr16:10788495-10789043 (-) & LTR & GAGAYC & - & Intronic & FAM18A (s) & - \\
\hline 45 & 17p13.1 & chr17:8056835-8057031 (+) & $\operatorname{LTR}^{\mathrm{b}}$ & not found & - & Intergenic & - & $A L O X 15 B$ \\
\hline 46 & 17p13.1 & chr17:8082291-8082848 (+) & LTR & CCAGG & - & Intronic & $A L O X 12 B$ (as) & MIR4314 \\
\hline 47 & $17 q 11.2$ & chr17:32913177-32913734 (+) & LTR & GGTATR & - & Intergenic & - & - \\
\hline 48 & $19 p 13.3$ & chr19:2863773-2863865 (+) & $L T R^{b}$ & not found & - & Intergenic & - & ZNF555; ZNF556 \\
\hline 49 & 19p13.2 & chr19:7100429-7100974 (-) & LTR & GTCTC & - & Intergenic & - & - \\
\hline 50 & $19 \mathrm{p} 12$ & chr19:21987441-21988020 (+) & LTR & ATAAYA & - & Intronic & ZNF208 (as) & - \\
\hline 51 & $19 \mathrm{p} 12$ & chr19:23876577-23877146 (-) & LTR & CTCCCC & - & Intergenic & - & - \\
\hline 52 & $19 q 13.11$ & chr19:34595165-34595718 (-) & LTR & TGTAGG & - & Intergenic & - & SCGBL \\
\hline 53 & $19 q 13.12$ & chr19:36636542-36637090 (-) & LTR & not found & - & Intergenic & - & ZNF382; ZNF461 \\
\hline 54 & $19 q 13.2$ & chr19:42667555-42668111 (-) & LTR & GTGTG & - & Intergenic & - & - \\
\hline 55 & $19 q 13.31$ & chr19:44283216-44283766 (-) & LTR & GTAAG & - & Intergenic & - & ZNF233; ZNF235 \\
\hline 56 & $19 q 13.32$ & chr19:46030891-46031467 (+) & LTR & CAAGGT & - & Intergenic & - & IGFL4; PGLYRP1 \\
\hline 57 & $19 q 13.41$ & chr19:51900477-51900716 (-) & $L_{T R}^{b}$ & not found & - & Intronic & ZNF649 (s); ZNF619 (as) & ZNF649-AS1 \\
\hline 58 & $19 q 13.41$ & chr19:52460866-52466497 (-) & Provirus $^{\mathrm{b}}$ & AAAAC & - & Intronic & ZNF578 (as) & ZNF534 \\
\hline 59 & $21 q 22.3$ & chr21:43698519-43699073 (+) & LTR & TTAG & - & Intergenic & - & RRP1B \\
\hline 60 & $21 q 22.3$ & chr21:43738447-43739417 (+) & LTR & CTAAT & AluSx; AluY & Intronic & PDXK (as) & - \\
\hline 61 & $22 q 11.21$ & chr22:20818927-20819480 (-) & LTR & TAAGA & - & Intronic & PIAKA (s) & - \\
\hline 62 & $22 q 13.31$ & chr22:45188740-45189285 (+) & LTR & TGCAAC & - & Intergenic & - & NUP50; KIAA0930 \\
\hline 63 & Xp11.23 & chrX:48047190-48047228 (-) & $\operatorname{LTR}^{\mathrm{b}}$ & not found & - & Intergenic & - & ZNF630-AS1 \\
\hline 64 & Xp11.22 & chrX:51636506-51637062 (+) & LTR & GCTCTA & - & Intergenic & - & - \\
\hline 65 & $X q 22.2$ & chrX:103469871-103470407 (-) & LTR & GRGGAG & - & Intergenic & - & - \\
\hline 66 & $\mathrm{Xq} 22.3$ & chrX:107232277-107232369 (-) & $\operatorname{LTR}^{\mathrm{b}}$ & not found & - & Intronic & PIH1D3 (as) & - \\
\hline 67 & Xq27.1 & chrX:139517757-139518302 (+) & LTR & CTTAAG & - & Intergenic & - & - \\
\hline 68 & Yq11.221 & chrY:12993871-13001093 (-) & Provirus & TGSATT & AluY; LTR2B & Intergenic & - & - \\
\hline 69 & Yq11.221 & chrY:13333756-13334302 (+) & LTR & CRYAGC & - & Intronic & UTY (as) & - \\
\hline 70 & Yq11.221 & chrY:16492757-16493302 (+) & LTR & TCCAAR & - & Intergenic & - & - \\
\hline
\end{tabular}

Chromosome bands and coordinates are according to the hg38 assembly [1] and RepeatMasker annotation [70]

As, antisense orientation; s, sense orientation; TSD, target site duplication; ${ }^{a}$ denotes a tandemly repeated LTR; ${ }^{b}$ denotes truncated elements.

Ambiguous nucleotides are indicated as follows: $\mathrm{K}, \mathrm{G}$ or T; M, A or C; S, C or G; R, A or G; W, A or T; Y, C or T

a common feature of present-day retroviruses as well [35]. Based on the published literature about HERV-W and HML-2, we compared the integration preferences of these two HERV families with HML-10 as well as all other HML families, except for HML-9 that was not annotated by RepeatMasker, at the genome-wide level. HML-10 sequences were found with higher frequency within the boundaries of RefSeq genes (47.94\%) than expected by random distribution (44.79\%), whereby sequences of HML-2 (28.76\%), HERV-W (27.95\%) and of all annotated HERV elements combined $(28.46 \%)$ were less abundant within genes (Fig. 1e). The intragenic sequence fractions of the other HML families were below the expected value for random integration and ranged between 24.35\% (HML-1) and $36.75 \%$ (HML-4). Thus, the frequent location of HML-10 within host genes appears to be a unique feature of this family and suggests an important and conserved function for gene regulation. The intronic HML-10 elements showed a distinct bias for integration in reverse orientation relative to the respective encompassing gene, with 20 being in reverse (antisense) and 7 in parallel (sense) orientation (Table 1). Two elements were reverse to one gene and parallel to another overlapping one. The integration bias of HML-10 indicates that the reverse orientation was evolutionarily favored, which is in line with previous findings of other HERV families [36-38]. One explanation for this observation is that parallel intronic proviruses are more likely to disrupt the encompassing gene due to the presence of transcription termination sites in the LTRs, which leads to negative selection of such integration events [39]. 


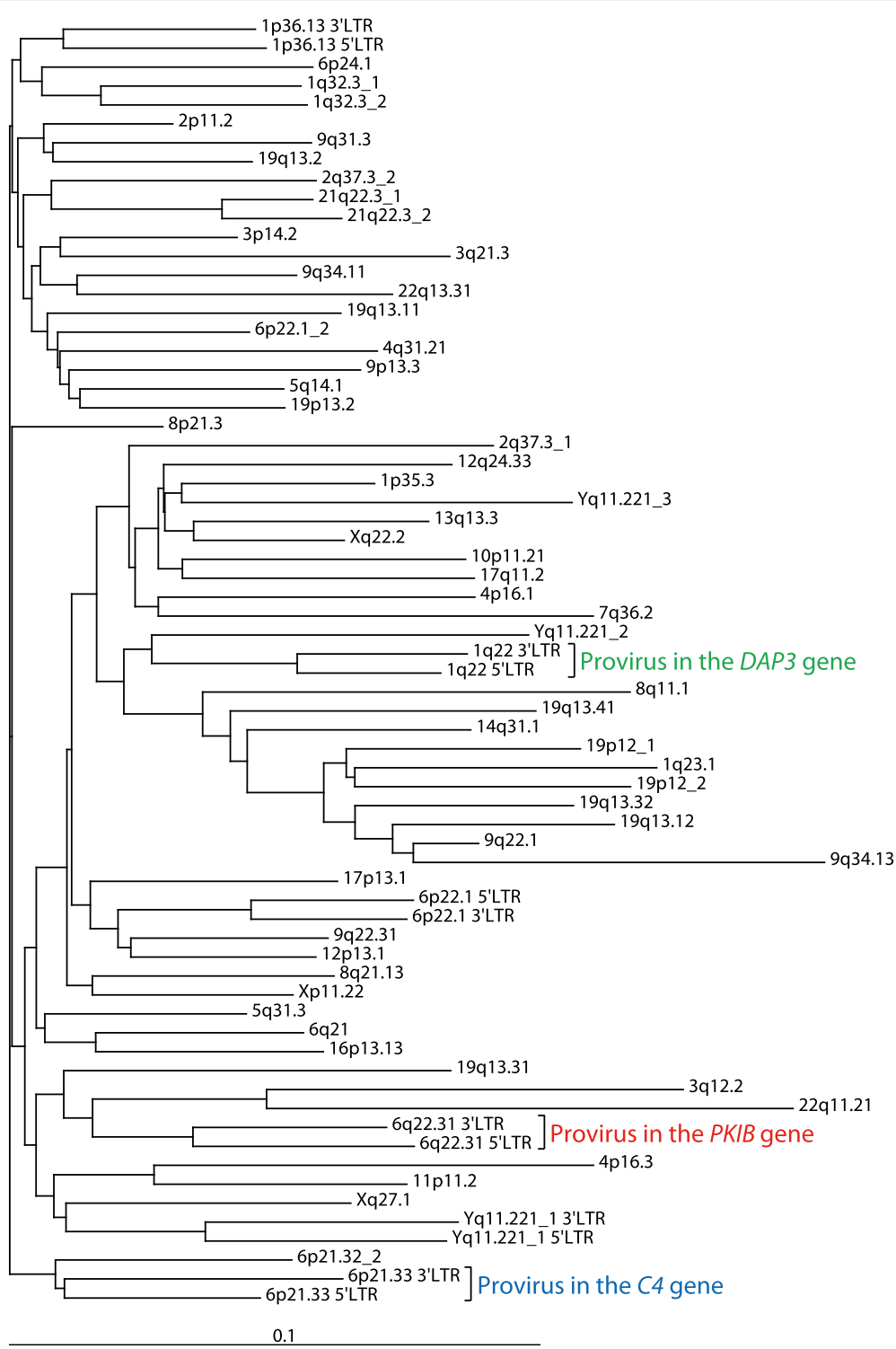

Fig. 2 Neighbor-joining tree of 68 complete HML-10 LTRs in the human genome. HML-10 LTR sequences (see Table 1) were retrieved from the human genome GRCh38/hg38 assembly [1] according to RepeatMasker [70] annotation. The horizontal bar represents 0.1 substitutions per nucleotide position

Contrarily, reverse oriented proviruses may even be beneficial by protecting from newly infecting retroviruses by antisense RNA mechanisms [19] and by contributing regulatory elements such as LTR promoters that can modulate gene expression in cis, as shown before $[7,14]$.

\section{HML-10 exerts differential LTR promoter activity in tumor cell lines}

To further investigate the potential of HML-10 in generating cis-regulatory transcripts, we determined LTR promoter activities of three complete proviruses located in reverse orientation within introns of host genes (Fig. 3). These were elements nos. 3, 22 and 25, within the
$D A P 3, C 4$ and PKIB (Protein kinase inhibitor beta) genes, respectively (Table 1 ). The PKIB gene harbors numerous other intronic HERV sequences not belonging to the HML-10 family that together with other REs constitute over $50 \%$ of its genomic sequence. Three additional HML-10 proviruses are located outside of genes, elements nos. 1, 20 and 68, and one found in an intron of the zinc finger protein gene ZNF578, no. 58, lacks the 5' LTR. We focused on the three complete and intronic proviruses, referred to as HML-10(DAP3), HML-10(C4) and HML-10(PKIB), that comprise six LTRs for promoter analysis, since these could potentially generate cis-regulatory transcripts. We preferred proviruses over 


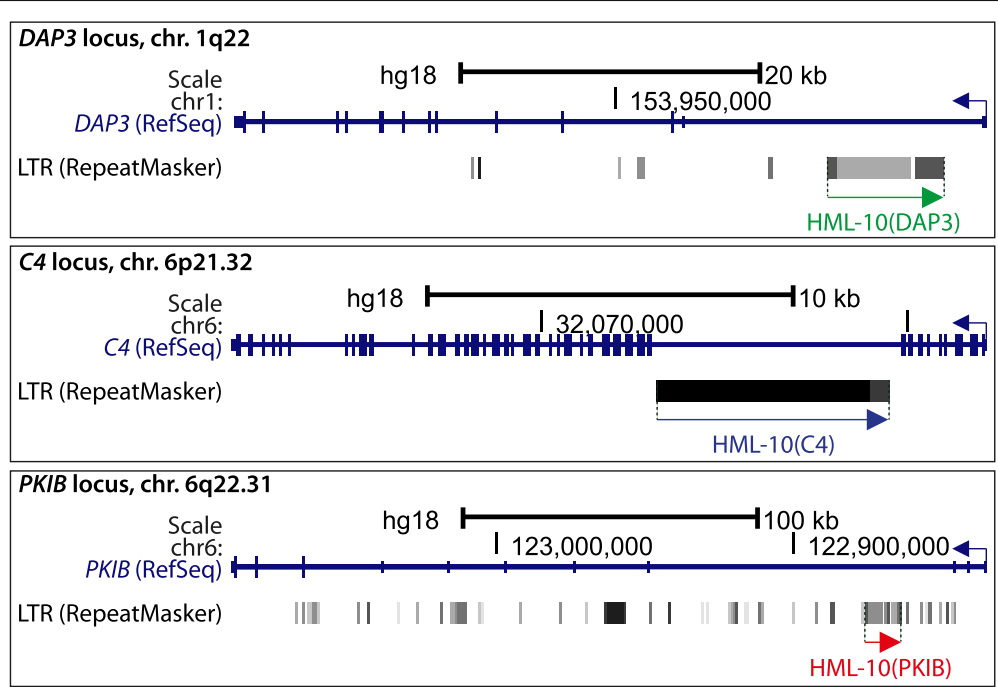

Fig. 3 Genomic organization of the HML-10(DAP3), HML-10(C4) and HML-10(PKIB) proviruses (from top to bottom). The grey rectangles in the LTR (RepeatMasker) track shows all annotated HERV elements including the indicated HML-10 proviruses. Images were retrieved and modified from the UCSC Genome Browser [68]

solitary LTRs since proviral LTRs of the related HML-2 and HERV-W families have been shown to be stronger promoters than the respective solitary LTRs $[8,15]$. We also found that the two LTRs of each HML-10 provirus clustered in the neighbor-joining tree (Fig. 2). Thus, despite their high sequence similarities these LTRs have resisted homologous recombination, suggesting their functional importance. HML-10 provirus RNA has been detected in various human tissues and cell lines by microarray analyses [22-27, 40-43] that, however, lack the information whether transcription is initiated in the 5'LTR or upstream of the provirus.

To assess their promoter activities, we cloned the LTRs of HML-10(DAP3), HML-10(C4) and HML-10(PKIB) into the promoter-free pGL3-Enhancer luciferase reporter vector, as described [19] (Fig. 4a). As HERV LTRs can be bidirectional promoters $[5,17,44]$, we also included the retroviral antisense orientation for each of the six LTRs. LTR promoter activity of HML-10(C4) has been demonstrated before with reporter assays in the human hepatocellular carcinoma cell line HepG2 and in COS7 monkey kidney cells [19]. Additionally, HML-10 pol transcripts have been identified in human hepatocellular carcinoma cells and in human embryonic kidney HEK293 cells by microarray analysis [26] (Table 2). Based on these findings, we transfected our pGL3-Enhancer constructs into HepG2 and HEK293T cells (HEK293 expressing SV40 virus Tantigen) to measure their promoter activities (Fig. 4b). The pGL3-Control vector bearing the SV40 promoter served as positive control and empty, promoter-free pGL3-Enhancer as negative control. HML-10(C4) showed significant transcriptional activity exclusively in the 3'LTR in HepG2 in both retroviral sense and antisense orientations. This is in accordance with a previous study that has demonstrated promoter activity in the $3^{\prime} \mathrm{LTR}$, but not in the $5^{\prime} \mathrm{LTR}$ of this provirus in the same cell line [19]. In HEK293T, we found transcription from the 5'LTR in retroviral sense orientation and from the 3'LTR in retroviral antisense orientation. HML-10(DAP3) exerted bidirectional promoter activity in its 5'LTR in both cell lines, whereas HML-10(PKIB) showed bidirectional promoter activity in its $3^{\prime}$ LTR, but in HEK293T only. Therefore, all three investigated proviruses showed transcriptional activity in at least one of their LTRs, with cell type-specific strength and orientation (Fig. 4b). While LTR promoter activity in retroviral antisense orientation was unlikely to primarily affect gene regulation, all three HERVs exerted promoter activity in retroviral sense orientation in one of their LTRs, which is antisense relative to the respective encompassing gene. Thus, the proviruses have the potential for antisense-mediated regulation of the encompassing DAP3, C4 and PKIB genes in cis in a cell type-specific manner.

Promoter activity of the HML-10(C4) 3'LTR has been reported previously to be suppressed by IFNY in HepG2 [19], which we reproduced (Fig. 4c). Likewise, the 5'LTR promoter of HML-10(DAP3) in retroviral sense orientation (antisense relative to the DAP3 gene) was dosedependently suppressed by IFN $\gamma$. We speculate this to be mediated by an IFN $\gamma$ activated site (GAS) matching the consensus motif $5^{\prime}$-TTNCNNNAA-3', a putative binding site for STAT1 homodimers that form during IFNY signaling [45]. This motif is present in all analyzed LTRs (Fig. 4d) as well as the SV40 promoter (data not shown). The latter is known to be inhibited by IFN $\gamma$ [46] and served as positive control for IFN $\gamma$-mediated suppression (Fig. 4c). In contrast, the herpes simplex virus 

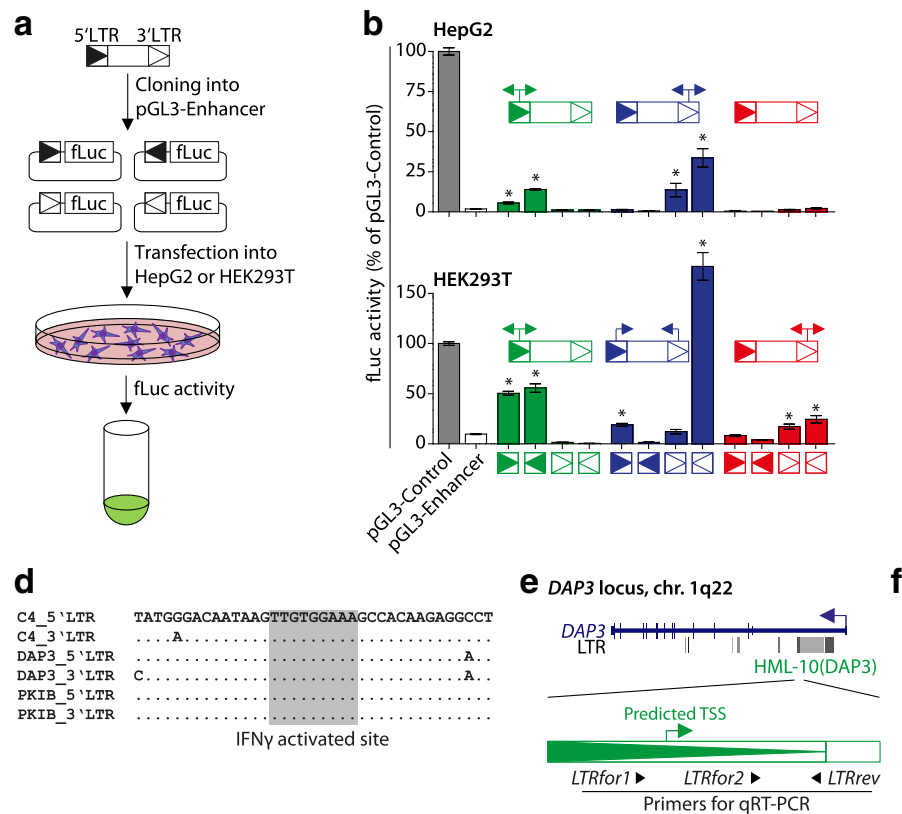
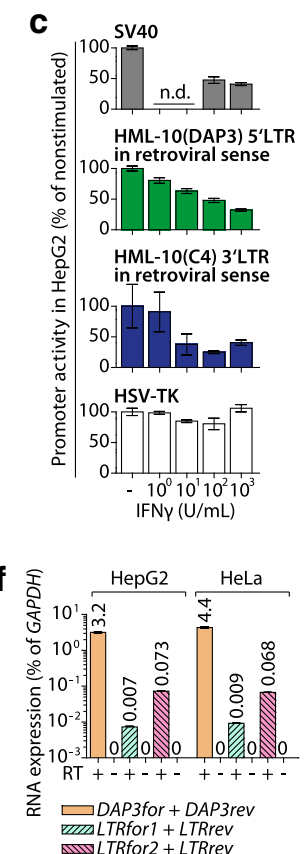

Fig. 4 Promoter activities of HML-10 LTRs. a LTRs of the HML-10(DAP3), HML-10(C4) and HML-10(PKIB) proviruses were cloned in both orientations into the promoter-free pGL3-Enhancer vector and transfected into HepG2 or HEK293T cells. Firefly luciferase (fLuc) activities were determined $24 \mathrm{~h}$ after transfection b Promoter activities expressed as fluc activity normalized to renilla luciferase (rLuc) activity of the co-transfected pGL4.74 vector in the indicated cell lines. The pGL3-Control vector bearing the SV40 promoter (grey bars) served as positive and empty pGL3-Enhancer (white bars) as negative control. Promoter activities were normalized to pGL3-Control set to 100\%. The bars show mean \pm SEM of three independent experiments in duplicates. ${ }^{*}$-value $\leq 0.05$, Student's $t$-Test compared to $\mathrm{pGL3-Enhancer.} \mathrm{c} \mathrm{For} \mathrm{HepG2} \mathrm{cells} \mathrm{the} \mathrm{effect} \mathrm{of} \mathrm{IFNy} \mathrm{stimulation} \mathrm{on} \mathrm{two} \mathrm{selected} \mathrm{LTRs} \mathrm{as} \mathrm{well}$ as the SV40 and HSV-TK promoters is shown. LTR and SV40 activity is expressed as fLuc normalized to rLuc signals, HSV-TK activity is expressed as rLuc activity only. The bars show mean \pm SEM of at least three independent experiments and were normalized to unstimulated (-) cells set to $100 \%$. n.d., not determined. $\mathbf{d}$ Identification of a conserved IFNy activated site (GAS) of the consensus sequence 5'-TTNCNNNAA-3' [45]. e Locations of primers used to detect transcripts originating from the $5^{\prime}$ LTR of HML-10(DAP3). The predicted TSS was identified as described in the text and Additional file 1: Figure S1. f Detection of DAP3 mRNA and HML-10(DAP3) transcripts in HepG2 and HeLa cells by qRT-PCR. cDNA samples prepared without reverse transcriptase (RT) for the indicated primer pairs, but with RT for GAPDH, served as controls. Values are normalized to GAPDH mRNA levels. Bars show mean \pm SD of two measurements. In most cases, the SD is too small to be visible

Table 2 Detection of HML-10(DAP3) pol transcripts by previously reported microarray studies [79]

\begin{tabular}{|c|c|c|c|}
\hline Tissue/cell type & HML-10(DAP3) RNA expressed & HML-10(DAP3) RNA not expressed & $\begin{array}{l}\text { HML-10(DAP3) RNA } \\
\text { variably expressed }\end{array}$ \\
\hline $\begin{array}{l}\text { Normal tissue and non-tumor } \\
\text { cell lines }(n=23)\end{array}$ & $\begin{array}{l}\text { Cervix [22], epidermal keratinocytes } \\
\text { (HaCaT) [26], thyroid [22], umbilical } \\
\text { vein endothelial cells (HUVEC) [40], } \\
\text { uterus [22] (5/23) }\end{array}$ & $\begin{array}{l}\text { Blood [22, 23], breast [25], colon [22], } \\
\text { heart [22], liver [22], lung [22], mamma } \\
{[22,23] \text {, neural stem cells (HNSC.100) }} \\
{[40], \text { ovary [22], placenta [22], prostate }} \\
{[22] \text {, rectum [22], skeletal muscle [22], }} \\
\text { stomach [22], testes [22] (15/23) }\end{array}$ & $\begin{array}{l}\text { Brain }[22,24] \text {, kidney } \\
{[22,27] \text {, skin }} \\
{[22,41](3 / 23)}\end{array}$ \\
\hline $\begin{array}{l}\text { Primary tumors and tumor } \\
\text { cell lines }(n=13)\end{array}$ & $\begin{array}{l}\text { CAKI (renal carcinoma) [26], GliNS1 } \\
\text { (neural tumor stem line) [40], HEK293 } \\
\text { (embryonic kidney) [26], HeLa } \\
\text { (cervix adenocarcinoma) [26], HuH-7 } \\
\text { (hepatocellular carcinoma) [26], } \\
\text { MIA PaCa-2 (pancreatic carcinoma) } \\
\text { [26], SK-N-MC (neuroblastoma) [42, 43], } \\
\text { SK-N-SH (neuroblastoma) [42], T47D } \\
\text { (ductal breast epithelial carcinoma) [26], } \\
\text { U-251MG (glioblastoma) [42] (10/13) }\end{array}$ & $\begin{array}{l}\text { Breast cancer [25], U-138MG } \\
\text { (glioblastoma) [42] (2/13) }\end{array}$ & $\begin{array}{l}\text { Renal cell carcinoma } \\
\text { [27] }(1 / 13)\end{array}$ \\
\hline
\end{tabular}


thymidine kinase (HSV-TK) promoter that was used to normalize promoter activities was unaffected by IFNY (Fig. 4c). The GAS motif is highly conserved among proviral HML-10 LTRs in the human genome (Fig. 4d) and the solitary LTRs (data not shown), which supports its functional relevance. Hence, IFN $\gamma$-mediated promoter suppression is likely a general feature of HML-10 LTRs, in line with the known antiviral activity of interferons [19]. This is of particular interest for possible HML-10mediated negative regulation of the encompassing genes, since mRNA expression of $C 4$ and DAP3 is known to be induced by IFN $\gamma[20,47]$ and $D A P 3$ is implicated in IFN $\gamma$-dependent apoptosis [20].

Based on our promoter activity studies, HML-10(DAP3) was the most interesting candidate for further investigation, since its 5 'LTR is the only one investigated that promoted transcription in the retroviral sense orientation, which is antisense to $D A P 3$, in both cell lines (Fig. 4b). The involvement of an HML-10(DAP3)-primed transcript in regulating the encompassing gene is suggested by the fact that DAP3 expression is induced [20], whereas the LTR promoter is suppressed by IFN $\gamma$ (Fig. 4c). In addition, HML-10(DAP3) RNA has been detected previously in various human cancer-derived cell lines but not in most healthy tissues (Table 2). This indicates a possible role in the regulation of DAP3 gene expression in cancer cells and some distinct tissues, including cervix, thyroid and uterus as well as epidermal keratinocytes and umbilical vein endothelial cells. Our promoter activity studies indicated that transcription of HML-10(DAP3) originated from the $5^{\prime}$ LTR (Fig. 4b). For further proof, we determined the most likely TSS within this promoter. Since LTR-dependent transcription relies on host RNA polymerase (RNA pol) II $[5,48]$ we sought to identify the two integral core elements of this promoter, Initiator (Inr) elements and TATA boxes [49]. The TSS within LTRs of the related HML-2 family has been identified previously within an Inr element with a TATA box about $10 \mathrm{bp}$ upstream of the Inr [50]. We identified a similar configuration a single time in the HML-10(DAP3) 5'LTR in retroviral sense orientation, an Inr element 11 bp downstream of a TATA box (Additional file 3: Figure S2). This Inr sequence contained the most likely TSS. We also identified a downstream promoter element (DPE) matching the consensus 5'-RGWYVT-3' sequence [49], a putative binding site for the transcription factor TFIID of the RNA pol II core promoter, at nucleotide position +19 relative to the putative TSS. To get experimental proof that HML10(DAP3) transcription is initiated within this putative TSS, we performed quantitative real-time PCR (qRT-PCR) measurements in HepG2 cells with a reverse primer located downstream of the TSS (LTRrev) and two different forward primers, one located upstream (LTRfor 1 ) and one downstream (LTRfor2) of the TSS (Fig. 4e and Additional file 3: Figure S2). If transcription was initiated from the TSS, we would expect higher expression measured using LTRfor 2 + LTRrev than with LTRfor $1+$ LTRrev primer combinations. This was indeed the case, whereby weak signals seen with LTRfor + LTRrev likely resulted from amplification of the intron of the DAP3 pre-mRNA (Fig. 4f). To avoid false signals from genomic DNA for these lowly abundant transcripts, we subjected the RNA preparations to DNase treatment prior to reverse transcription and included control samples without reverse transcriptase that did not result in detectable amplification. We thus verified expression of the HML-10(DAP3) RNA that is present at about 40-fold lower levels than the $D A P 3$ mRNA, and provide further evidence that it originates from the 5'LTR around the predicted TSS. These findings confirmed the weak but significant transcription of this LTR in the promoter activity studies in retroviral sense orientation in the same cell line (Fig. 4b). Our findings are in agreement with reported microarray data that demonstrated expression of the retroviral transcript in various cell lines, which extends into the pol gene of the HML-10(DAP3) provirus (Table 2). However, although the primer combinations were designed to only amplify the HML-10(DAP3) sequence, as judged by in silico PCR analysis, we cannot completely rule out that transcripts of other potentially active HML-10 elements were coamplified.

The pro-apoptotic effect of DAP3 has been described previously in HeLa cells [20] in which HML-10(DAP3) RNA has been identified by microarray analysis (Table 2). Accordingly, we detected HML-10-primed transcripts by qRT-PCR in HeLa where it was present at comparable levels as in HepG2 (Fig. 4f). We therefore selected HeLa cells to determine the functional relevance of HML10(DAP3) RNA on the expression of DAP3.

\section{Inactivation of HML-10(DAP3) RNA induces DAP3 expression and apoptosis in HeLa cells}

Having confirmed the presence of HML-10(DAP3) RNA in HeLa cells and its likely origin within the proviral 5' LTR, we sought to determine its function within the cell. We expected the retroviral RNA to suppress DAP3 gene expression in cis similar to the previously described LTR-primed regulatory transcripts $[7,14]$. To determine its potential regulatory function we aimed at inactivating the retroviral RNA by means of sequence-specific ASOs. We opted for ASOs rather than siRNAs that are both known to be active in the nucleus [51,52], the common site of action of LTR-primed transcripts [5], as siRNAs may directly influence DAP3 expression levels through the passenger strand that would be antisense to the DAP3 pre-mRNA. ASO-mediated inactivation of the HML-10(DAP3) RNA was expected to activate DAP3 gene expression. 
We designed four ASOs downstream of the putative TSS, ASOs 1-4, to counteract the retroviral RNA (Fig. 5a). At $24 \mathrm{~h}$ after transfecting the ASOs at 25 or 50 nM into HeLa cells, we determined HML-10(DAP3) and $D A P 3$ expression at the RNA level by qRT-PCR. Transfecting the ASOs caused an increase in DAP3 mRNA levels, as expected, but not a decrease in HML-10(DAP3) RNA (Fig. 5b). These observations likely indicate that the ASOs blocked association of DAP3 pre-mRNA with the retroviral RNA, but did not significantly mediate cleavage of the latter. Although RNase $\mathrm{H} 1 / \mathrm{H} 2$-dependent hybridspecific RNA degradation has been reported to be induced by ASOs [51, 53], cleavage efficiency is largely sequencedependent and the HML-10(DAP3) RNA might resist degradation. For these reasons, measuring DAP3 mRNA levels was the only feasible way to assess the impact of inactivating the retroviral RNA. Transfecting ASOs 1-4 resulted in increased $D A P 3$ mRNA levels with varying efficiencies (Fig. 5b). When used at $25 \mathrm{nM}$, ASOs 1-4 increased DAP3 mRNA levels about 5-fold as compared to non-transfected control cells. The most efficient ASO 2 exerted a dose-dependent increase of the DAP3 mRNA up to 10 -fold at $50 \mathrm{nM}$. Both control ASOs, one with a random sequence (Mock) and one immediately upstream of the 5'LTR, did not significantly change DAP3 expression levels, demonstrating a sequence-dependent effect and that the HML-10(DAP3) RNA originates from the 5' LTR. Although ASOs 1-4 were designed to only map to the DAP3 locus, we consider the possibility that HML-10 RNA species transcribed at other loci that may act in trans on DAP3 expression may have been inactivated by these ASOs as well. Overall, the use of ASOs to counteract HML-10-primed transcripts confirmed their negative impact on DAP3 mRNA expression levels.

DAP3 is an adapter protein that links the intracellular portion of DRs to the Fas-Associated Death Domain (FADD) in the DR pathway of extrinsic apoptosis [21]. Consequently, we expected the HML-10(DAP3) RNA to suppress apoptosis via this pathway. Overexpressing $D A P 3$ has been shown to induce apoptosis in HeLa cells [20]. We wondered whether upregulation of $D A P 3$ by the most effective ASO 2 at $50 \mathrm{nM}$ (Fig. 5b) was a

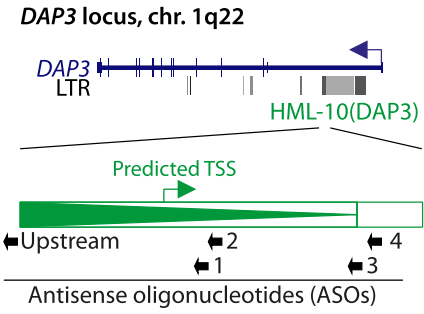

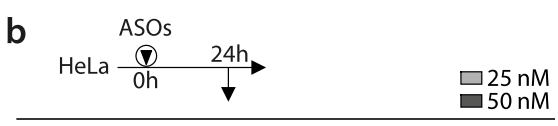
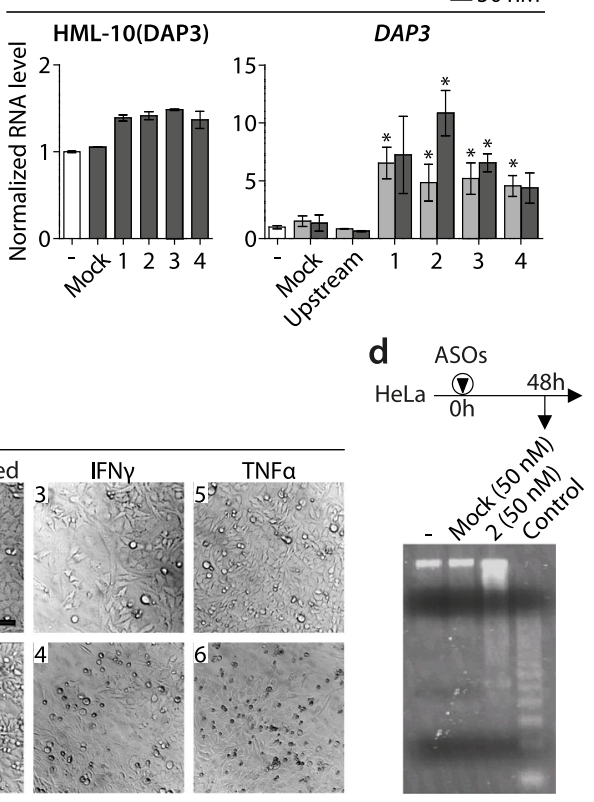

Fig. 5 Inactivating the HML-10(DAP3) RNA induces DAP3 expression and apoptosis in HeLa cells. a Target regions of sequence-specific ASOs are indicated. ASOs 1-4 are in antisense orientation to the retroviral transcript and in sense orientation to the DAP3 transcript. The ASO designated as Upstream served as control. b Cells were transfected with 25 or $50 \mathrm{nM}$ of the indicated ASOs. At $24 \mathrm{~h}$ after transfection, expression levels of HML-10(DAP3) (left) and DAP3 mRNA (right) were determined by qRT-PCR. Bars show mean \pm SEM of three independent experiments. RNA levels were normalized to GAPDH and levels of non-transfected cells were set to $1 .{ }^{*} P$-value $\leq 0.05$, Student's $t$-Test against Mock. c Cells were transfected with the indicated ASOs at $50 \mathrm{nM}$, after $24 \mathrm{~h}$ stimulated with $1000 \mathrm{U} / \mathrm{mL}$ IFNy or $100 \mathrm{ng} / \mathrm{mL}$ TNFa, or left unstimulated. After additional $24 \mathrm{~h}$, Trypan Blue exclusion as indicator of dead cells (left), MTS cell viability assays (center) or light microscopic analysis (right) was performed. Bars show mean \pm SEM of three independent experiments in duplicates. ${ }^{*}$-value $\leq 0.05$, Student's $t$-Test. The scale bar in light microscopy panel 1 is $100 \mu \mathrm{m}$. $\mathbf{d}$ Cells were transfected with the indicated ASOs at $50 \mathrm{nM}$. At $48 \mathrm{~h}$ after transfection, genomic DNA of these cells was prepared with the Apoptotic DNA Ladder Kit (Roche). The control DNA is from apoptotic U937 cells provided with the kit 
sufficient to cause apoptosis. To this end, we compared the effect of ASO 2 with known apoptosis-inducing stimuli, tumor necrosis factor-alpha (TNF $\alpha)$ and IFN $\gamma$, on HeLa cells. Both cytokines significantly induced cell death associated with diminished cell viability, as well as cell rounding characteristic of apoptosis (Fig. 5c). Likewise, HeLa cells transfected with ASO 2 showed similar signs of apoptosis but not those transfected with Mock ASO. The fraction of dead cells was significantly higher for ASO 2-transfected cells when compared to Mocktransfected cells $\left(24.8 \%\right.$ vs. $\left.8.0 \%, P=10^{-4}\right)$, and cell viability was lower ( $47.9 \%$ vs. $76.8 \%$ relative to non-transfected cells, $P=10^{-4}$ ). In addition, transfection of ASO 2, but not of Mock, induced features of apoptosis, such as detachment from the tissue culture dish, rounding and shrinking (Fig. 5c, light microscopy panels 1 and 2). This was supported by another test for apoptosis, genomic DNA fragmentation, that occurred upon transfection of ASO 2 (Fig. 5d). These findings provided evidence that ASO 2mediated induction of DAP3 mRNA led to increased expression of DAP3 protein that is required for apoptosis and DNA fragmentation. Thus, inactivating HML-10(DAP3) RNA increased DAP3 expression sufficiently to induce apoptosis, which demonstrates the functional relevance of this retroviral transcript.

In parallel, we assessed whether inactivating HML10(DAP3) RNA also increased the susceptibility to apoptosis by TNF $\alpha$. We expected this since TNF $\alpha$ stimulates extrinsic apoptosis via the DR pathway that involves DAP3 [21]. Thus, inactivation of HML-10(DAP3) RNA with resulting DAP3 overexpression and TNF $\alpha$ stimulation may promote apoptosis synergistically. Indeed, ASO 2-transfected HeLa cells that were additionally stimulated with TNF $\alpha$ exhibited increased signs of apoptosis compared to unstimulated ASO 2-transfected cells (Fig. 5c, light microscopy panels 2 and 6), and contained a larger fraction of dead cells (38.1\% vs. $24.8 \%$ ), albeit without statistical significance (Fig. 5c). Stimulating ASO 2transfected cells with IFN $\gamma$ had less pronounced effects on the fraction of dead cells and viability (Fig. 5c), which may be because IFN $\gamma$ induces apoptosis independent of DR signaling. Conclusively, we showed that $D A P 3$ expression is negatively regulated by the HML10(DAP3) RNA to an extent that apoptosis is inhibited in HeLa cells.

\section{Discussion}

Here we have characterized the previously little described HML-10 family of endogenous retroviruses in the human genome and studied its potential in regulating host gene expression. We found that the infectious progenitor of HML-10 invaded the ancestral genome of OWMs about 35 Mya (Fig. 1b). With 70 identified elements, HML-10 is a relatively small HERV family when compared, for instance, to the intensely investigated HML-2 that constitutes about 2500 sequences in the human genome [4]. It is known that HERVs, after de novo integration, may increase in number due to chromosomal duplication events [4]. However, sequence comparisons of the TSDs (Table 1) and the flanking regions (Additional file 1: Figure S1) indicated that only one of the 70 identified HML-10 elements is the result of a chromosomal duplication, whereas the other 69 elements most likely arose by independent retroviral integrations. We found an unusually high abundance of HML-10 within introns of host genes when compared to other HERV sequences including those of phylogenetically related HML families (Fig. 1e), indicating that this family in particular has been evolutionary co-opted for gene regulatory functions. Since LTR promoter activity of the provirus in the $C 4$ gene has been demonstrated previously [19], we hypothesized HML-10 to express LTRprimed regulatory transcripts in cis similar to recently reported HML-2 [14] and HERV-E [7] LTRs.

To assess their potential in expressing such regulatory RNAs, LTRs of three selected, intron-located HML-10 proviruses were subjected to promoter activity studies in HepG2 and HEK293T cells (Fig. 4b). Interestingly, both strength and orientation of LTR transcription differed substantially between the cell lines. Based on the promoter activity studies, all three investigated HML-10 proviruses had the potential to negatively regulate their encompassing genes by priming antisense RNAs. The HML-10(DAP3) provirus located in the DAP3 gene showed LTR promoter activity in retroviral sense orientation (antisense relative to DAP3) in both cell lines and was therefore selected for further analysis (Fig. 4b). DAP3 is a signaling protein involved in the DR pathway of extrinsic apoptosis that induces apoptosis when overexpressed [20, 21]. Promoter activity of the HML10(DAP3) 5'LTR in retroviral sense orientation (antisense relative to the DAP3 gene) was suppressed by IFN $\gamma$, as reported previously for the HML-10 provirus in the C4 gene [19] (Fig. 4c). This might, at least partially, explain how DAP3 gene expression is induced by IFNY [20]. In HeLa cells, we found that counteracting the retroviral transcript by sequence-specific ASOs led to an increase of DAP3 expression levels sufficient to induce apoptosis (Fig. 5b,c). Two control ASOs, one targeting a region upstream of and one with a randomized sequence, did neither induce DAP3 mRNA expression nor apoptosis, verifying that the ASO transfection procedure itself did not exert any non-specific effects on these two read-outs. Thus, the HML-10(DAP3) RNA suppressed apoptosis in HeLa. HML-10 transcripts originating from other loci may have been inactivated by the ASOs as well and consequently might also contribute to the reduction of DAP3 expression in trans. ASO-mediated inactivation confirmed that the HML-10-primed transcripts, 
despite being about 60-fold weaker expressed than the DAP3 mRNA in this cell line (Fig. 4f), had a substantial impact on DAP3 expression levels. Indeed, regulatory non-coding RNAs are often weakly expressed [54] and capable of substantially down-regulating gene expression even if 10-100 fold less abundant than their respective mRNA [55]. Among the mechanisms that have been proposed for this kind of gene regulation is the induction of repressive epigenetic modifications that lead to heterochromatin formation, or transcriptional collision of opposing RNA polymerases [54]. It has been shown previously that preventing association between weakly expressed regulatory RNAs and their corresponding mRNA (as opposed to degradation of the regulatory RNA) is sufficient to substantially induce mRNA expression [55], which might explain why we did not observe ASO-mediated degradation of HML-10 RNA but nevertheless an increase in DAP3 mRNA expression levels (Fig. 5b).

Promoter activity studies (Fig. 4b), qRT-PCR experiments (Fig. 4f), and the fact that the ASO immediately upstream of the LTR did not affect DAP3 expression levels (Fig. 5b) provided evidence that the retroviral RNA originates from the proviral 5'LTR. We determined the most likely TSS within this LTR by in silico sequence analysis (Additional file 3: Figure S2). Attempts to experimentally verify this TSS by 5 'RACE-PCR as described previously [14] were not successful, as orientation-specific cDNA synthesis did not yield sufficient starting material for subsequent PCR reactions (see Methods section for details). Insufficient orientation-specific cDNA synthesis may have been due to the low abundance of the HML10(DAP3) RNA as seen by qRT-PCR (Fig. 4f) and is a known issue with rare transcripts [56]. Thus, the actual TSS of the retroviral transcript may differ from the predicted one but our experiments provide evidence that it is located between the target regions of ASO upstream and ASO 1 (Fig. 5a). Our findings indirectly confirmed the expression of HML-10(DAP3) RNA in HeLa cells, which was supported by reported microarray experiments (Table 2). Further direct proof could be obtained by sequencing cDNA clones and identifying genomic markers that are unique to the HML-10(DAP3) copy, such as the $A l u S p$ or MER11B repeats that are integrated into this provirus (Table 1).

Suppression of apoptosis, as mediated by the HML10(DAP3) RNA in HeLa cells, is a general hallmark of cancer cells [57]. Thus, retroviral transcripts may contribute to the malignant cellular phenotype of this cell line by counteracting $D A P 3$ expression, and thereby suppressing apoptosis. Aberrant expression levels of DAP3 have been suggested to play a role in some cases of malignant disease [58-63]. The data shown here indicate that most of the HML-10 LTRs are even weaker expressed than the one analyzed. We hypothesize that the LTRs, which are normally strong promoters in infectious retroviruses, have been silenced by mutation during evolution. Thus, they likely play a limited role in cancer promotion.

The data presented in Fig. 4b-c suggests that the expression of LTR-primed transcripts varies substantially in intensity and direction depending on the cell type as well as the action of cytokines. Moreover, despite its weak expression at about 60-fold lower levels than DAP3 mRNA (Fig. 4f) the HML-10-primed RNA had a strong impact on DAP3 gene regulation (Fig. 5b). Consistent contributions of this and other HERV-primed RNAs to various tissues or tumors may therefore be hard to identify. However, the presence of HML10(DAP3) RNA in many tumor cell lines and the absence in most healthy tissues (Table 2) suggest that its upregulation may be a relevant feature in some human cancer diseases. This is in line with the observation that transcriptional activation of HERVs and other REs by epigenetic DNA demethylation is a frequent characteristic of malignant cells [64-66].

\section{Conclusions}

This work provides experimental support for recent evidence that HERVs and other REs play a role in gene regulation and cellular processes relevant to mammalian tumor cell formation. In the case presented here, transcripts of the previously little described HML-10 family suppressed the pro-apoptotic DAP3 gene and consequently, apoptosis in HeLa cells. Therefore, we could verify a direct link between HERV expression and cellular phenotype in this cell line. A potential role of these LTRs in promoting a malignant phenotype possibly by inducing resistance to apoptosis as described here in other cell lines or tissues requires further investigation.

\section{Methods}

Identification of HML-10 elements in the human genome The Table Browser function [67] of the UCSC Genome Browser [68] was used to identify HML-10 elements in the human genome. We queried the Repbase sequence of HML-10 LTRs, LTR14 [69], in the RepeatMasker track [70] of the GRCh38/hg38 human genome assembly [1]. This search yielded 86 hits. Manual inspection of these hits revealed the 70 unique HML-10 elements listed in Table 1.

\section{Estimation of the evolutionary age of HML-10 proviruses}

For each of the six complete HML-10 proviruses (elements Nos. 1, 3, 20, 22, 25 and 68 in Table 1), both LTR sequences ( $5^{\prime}$ and $3^{\prime}$ LTRs) were aligned with Clustal X 2.0 [71]. The evolutionary age of each provirus was calculated from the number of mutations between both 
LTRs by applying an estimated nucleotide substitution rate of 2.28 per site and year $\times 10^{-9}$ as described [28].

\section{Construction of phylogenetic neighbor-joining trees}

The $\mathrm{pol}$ sequences of HML-10 and other betaretroviruses were retrieved from published literature $[18,72]$. The fasta protein sequences can be found in Additional file 4. Sequences were aligned with Clustal X 2.0 [71] using standard parameters of the Multiple Alignment Mode. The neighbor-joining tree was visualized with TreeView 1.6.6 [73]. The phylogenetic tree of HML-10 LTR nucleotide sequences and those of the flanking sequences shown in Additional file 1: Figure S1 were constructed similarly. All nucleotide sequences were retrieved from the UCSC Genome Browser [68] and the current release of the human genome, GRCh38/hg38 [1].

\section{Identification of target site duplications}

The sequences immediately up- and downstream of RepeatMasker-annotated HML-10 elements (Table 1) were searched for homologous sequences in the retroviral sense orientation. Homologous sequences of at least $5 \mathrm{bp}$ were defined as TSDs, allowing for one (5 bp TSDs) or two (6 bp TSDs) nucleotide mismatches.

\section{Location of HERV sequences relative to human genes}

The fractions of intragenic HERV sequences were determined with the UCSC Table Browser [67] using the GRCh38/hg38 human genome assembly [1]. HERV elements were identified as described below in this paragraph within the RepeatMasker track [70]. The output of these searches was used to generate custom tracks covering the sequences of the respective HERV families. Using the intersection function, the overlap of HERV sequences with a custom track representing full-length RefSeq genes was determined, yielding the following values (displayed as: HERV family, Repbase annotation, sequence covered, sequence intersected with RefSeq genes): HML-1, LTR14A / LTR14B / LTR14C, 274,910 bp, 66,940 bp (24.35\%); HML2, LTR5A / LTR5B, 595,281 bp, 171,219 bp (28.76\%); HML-3, MER9B / MER9a1 / MER9a2 / MER9a3, 568,179 bp, 151,429 bp (26.65\%); HML-4, LTR13 / LTR13A, 545,702 bp, 200,556 bp (26.75\%); HML-5, LTR22 / LTR22A / LTR22B / LTR22B1 / LTR22B2 / LTR22C / LTR22CO / LTR22C2 / LTR22E, 396,533 bp, 105,855 bp (26.70\%); HML-6, LTR3 / LTR3A / LTR3B, 130,701 bp, 37,058 bp (28.35\%); HML-7, MER11D, 194,536 bp, 60,756 bp (31.23\%); HML-8, MER11A / MER11B / MER11C, 2,222,448 bp, 656,281 bp (29.53\%); HML-10, LTR14, 40,556 bp, 19,443 bp (47.94\%); HERV-W, LTR17, 482,257 bp, 134,803 bp (27.95\%). All RepeatMaskerannotated HERV elements covered 266,970,452 bp, of which $75,967,800$ bp (28.46\%) intersected with RefSeq genes. The fraction of the total genome
$(3,088,269,808 \mathrm{bp})$ accounted for RefSeq genes was $1,320,982,363$ bp (44.97\%).

\section{Cell lines and culture conditions}

HeLa (ATCC CCL-2), HepG2 (ATCC HB-8065) and HEK293T cell lines were cultivated in complete growth medium; Dulbecco's Modified Eagle's Medium (DMEM) (Invitrogen, Carlsbad, CA, USA) supplemented with 10\% heat-inactivated fetal calf serum (Invitrogen) and $100 \mathrm{U} /$ $\mathrm{mL}$ penicillin, $100 \mu \mathrm{g} / \mathrm{mL}$ streptomycin and $0.25 \mu \mathrm{g} / \mathrm{mL}$ amphotericin (Antibiotic-Antimycotic by Invitrogen). Cells were incubated at $37{ }^{\circ} \mathrm{C}$ with $5 \% \mathrm{CO}_{2}$. Subcultivation ratios ranged between 1:2 and 1:10.

\section{Primers}

All primers were synthesized by Microsynth AG, Balgach, Switzerland. Primer sequences are listed in Additional file 5. Primer sequences were designed such that they only amplified the desired regions, as verified by the in silico PCR analysis tool of UCSC on https://genome.ucsc.edu/ cgi-bin/hgPcr/.

Construction of $\mathrm{pGL} 3$-Enhancer luciferase reporter vectors LTRs of HML-10(C4), HML-10(DAP3) and HML-10(PKIB) were amplified by standard PCR from genomic DNA of the QBL cell line (No. 4070713) obtained from the Health Protection Agency Culture Collections (ECACC, Salisbury, UK), using primer pairs with a HindIII or XhoI cleavage site at their $5^{\prime}$ ends. HML-10(C4) primer pairs: 5'LTR(s), C4_5LTRforHindIII + C4_5LTRrevXhoI; 5'LTR(as), C4_5L TRforXhoI + C4_5LTRrevHindIII; 3'LTR(s), C4_3LTRforHin dIII + C4_3LTRrevXhoI; 3'LTR(as), C4_3LTRforXhoI + C4_3 LTRrevHindIII. HML-10(DAP3) primer pairs: 5'LTR(s), DAP3_5LTRforHindIII + DAP3_5LTRrevXhoI; 5'LTR(as), D AP3_5LTRforXhoI + DAP3_5LTRrevHindIII; 3'LTR(s), DA P3_3LTRforHindIII + DAP3_3LTRrevXhoI; 3'LTR(as), DAP 3_3LTRforXhoI + DAP3_3LTRrevHindIII. HML-10(PKIB) primer pairs: 5'LTR(s), PKIB_5LTRforHindIII + PKIB_5 LTRrevXhol; 5'LTR(as), PKIB_5LTRforXhoI + PKIB_5LTRrevHindIII; 3'LTR(s), PKIB_3LTRforHindIII + PKIB_3LTRrevXhoI; 3'LTR(as), PKIB_3LTRforXhoI + PKIB_3LTRrev HindIII). Cycling conditions were $10 \mathrm{~min} .95^{\circ} \mathrm{C}$; $\left(30 \mathrm{s.} 95^{\circ}\right.$ C, 30 s. $60{ }^{\circ} \mathrm{C}, 30$ s. $\left.72{ }^{\circ} \mathrm{C}\right) \times 40 ; 7 \mathrm{~min} .72{ }^{\circ} \mathrm{C}$. LTRs were cloned into the pGL3-Enhancer vector (Promega, Madison, WI, USA), containing the fLuc gene as reporter, after digestion with HindIII and XhoI (New England Biolabs, Ipswich, MA, USA) and phosphatase treatment. Vectors were ligated with T4 DNA Ligase (New England Biolabs). All vector constructs were heat-shock-transformed into competent E. coli JM109 (Promega). Positive colonies were detected by ampicillin resistance on selective agar plates. Selected clones were grown in ampicillin-containing LB medium and plasmid DNA was isolated with the QIAamp Plasmid DNA Mini Kit (Qiagen, Hilden, Germany). 
Plasmid DNAs were screened for correct inserts by restriction enzyme digestions using appropriate enzyme combinations and subsequent agarose gel electrophoresis as well as by capillary sequencing (Microsynth, Balgach, Switzerland).

\section{Determination of LTR promoter activities}

Freshly passaged HepG2 or HEK293T cells were seeded into 24-well tissue culture plates $\left(4 \times 10^{4}\right.$ cells per well in complete growth medium) and cultivated overnight to $\sim 80 \%$ confluence. Cells were transfected with $50 \mathrm{ng} /$ well of pGL3-Enhancer constructs, empty pGL3-Enhancer, or pGL3-Control, $4 \mathrm{ng} /$ well of pGL4.74 vector for normalization (Promega) and $346 \mathrm{ng} /$ well of unrelated carrier DNA using DreamFect Gold transfection reagent (OZ Biosciences, Marseille, France) following the manufacturer's recommendations. Vector pGL4.74 contains the renilla luciferase (rLuc) gene under control of the herpes simplex virus thymidine kinase (HSV-TK) promoter. Medium was replaced with fresh prewarmed complete growth medium $6 \mathrm{~h}$ post-transfection. At $24 \mathrm{~h}$ post-transfection, medium was aspirated, cells were rinsed with prewarmed PBS, lysed, and fLuc and rLuc activities in each sample were determined with the DualGlo Luciferase Assay System (Promega) in a Sirius Luminometer (Berthold Detection Systems, Pforzheim, Germany). fLuc activities were normalized to rLuc activities for each sample. To assess the effect of IFNY stimulation on promoter activities, selected pGL3-Enhancer constructs were transfected into HepG2 cells as above and were stimulated with different amounts of recombinant human IFN $\gamma$ (PeproTech, Rocky Hill, NJ, USA) by addition to the growth medium immediately after medium change $6 \mathrm{~h}$ post-transfection. fluc activities were determined $30 \mathrm{~h}$ post-transfection.

\section{Inactivation of HML-10(DAP3) RNA with ASOs}

The ASOs were 25-mer DNA molecules with phosphorothioate bonds at the flanking three nucleotides on both sides to confer exonuclease resistance. ASOs for inactivating the HML-10(DAP3) RNA were designed to be complementary to regions within the $5^{\prime}$ LTR or the proviral body downstream of the predicted TSS. We used only sequences that uniquely mapped to their respective target region and nowhere else in the human genome. A Mock ASO with a randomized sequence and one complementary to a region shortly upstream of the 5'LTR were used as negative controls. ASOs were purchased from Microsynth. Their sequences are listed in Additional file 6.

\section{qRT-PCR}

Freshly passaged HepG2 or HeLa cells were seeded in 96-well plates $\left(10^{4}\right.$ cells per well in complete growth medium) and grown overnight to $\sim 80 \%$ confluence. Cells were transfected with 25 or $50 \mathrm{nM}$ of the indicated
ASOs using DreamFect Gold transfection reagent (OZ Biosciences) according to the manufacturer's recommendations. Medium was replaced with fresh prewarmed complete growth medium $6 \mathrm{~h}$ post-transfection. At $24 \mathrm{~h}$ post-transfection, total RNA was extracted using the QIAamp RNA Blood Mini Kit (Qiagen), including an on-column DNA digestion step with the RNase-free DNase Set (Qiagen). First strand cDNA was synthesized using the High Capacity cDNA Reverse Transcription Kit (Applied Biosystems, Foster City, CA, USA) with random hexamer primers. qRT-PCR was performed using the TaqMan Universal PCR Master Mix (Applied Biosystems) with the addition of 1:10000 (v/v) SYBR Green I (Sigma-Aldrich, St. Louis, MO, USA) and primers specific for DAP3 (DAP3for +DAP3rev) or GAPDH (GAPDHfor + GAPDHrev) mRNAs, HML10(DAP3) RNA (LTRfor2 + LTRrev) or LTRfor1 + LTRrev as control reaction. Cycling conditions were $2 \mathrm{~min} .50^{\circ}$ C; 10 min. $95^{\circ} \mathrm{C}$; $\left(15\right.$ s. $\left.95^{\circ} \mathrm{C}, 1 \mathrm{~min} .58{ }^{\circ} \mathrm{C}\right) \times 65$. Specificity of the PCR reactions was assessed by checking for correct amplicon lengths and amplification artifacts by agarose gel electrophoresis. All shown RNA levels were calculated by relative quantification (double delta $\mathrm{Ct}$ method) using GAPDH as reference, with primer efficiencies calculated from serial dilutions of HepG2 cDNA samples. Control samples without addition of reverse transcriptase gave no amplification signals.

\section{Strand-specific CDNA synthesis}

A number of $10^{6}$ freshly passaged HepG2 or HeLa cells were seeded into wells of 6-well plates and grown overnight to $\sim 80 \%$ confluence. Total RNA was extracted using the QIAamp RNA Blood Mini Kit (Qiagen). First strand cDNA was synthesized using either the Reverse Transcriptase of the High Capacity cDNA Reverse Transcription Kit (Applied Biosystems), or the Thermoscript Reverse Transcriptase (Invitrogen) with primers specific for the HML-10(DAP3) transcript (Additional file 5). Different incubation times and temperatures (ranging from 25 to $60{ }^{\circ} \mathrm{C}$ ) were evaluated. To assess reverse transcription efficiencies, qRT-PCR was done using TaqMan Universal PCR Master Mix (Applied Biosystems) with the addition of 1:10000 (v/v) SYBR Green I (Sigma-Aldrich) and primers LTRfor $2+$ LTRrev. Cycling conditions were $2 \min .50^{\circ} \mathrm{C}$; $10 \mathrm{~min} .95^{\circ} \mathrm{C}$; $\left(15 \mathrm{s.} 95^{\circ} \mathrm{C}, 1 \mathrm{~min} .58^{\circ} \mathrm{C}\right) \times 65$. No specific amplification was detected, while positive controls with cDNA prepared with random hexamer primers and with genomic human DNA yielded HML-10(DAP3)specific amplicons.

\section{Trypan Blue exclusion and cell viability (MTS) assays}

Freshly passaged HeLa cells were seeded in 48-well plates ( $2 \times 10^{4}$ cells per well in complete growth medium) and cultivated overnight to $\sim 70 \%$ confluence. Cells were transfected 
with $50 \mathrm{nM}$ of the indicated ASOs using the DreamFect Gold transfection reagent (OZ Biosciences) according to the manufacturer's recommendations. Medium was replaced with fresh prewarmed complete growth medium $6 \mathrm{~h}$ posttransfection. At $24 \mathrm{~h}$ post-transfection, cells were stimulated with $1000 \mathrm{U} / \mathrm{mL}$ recombinant human IFNY (PeproTech) or $100 \mathrm{ng} / \mathrm{mL}$ recombinant human TNF $\alpha$ (Biomol, Hamburg, Germany) for $24 \mathrm{~h}$ by addition to the growth medium, or left unstimulated. For Trypan Blue exclusion assays, cells were harvested $48 \mathrm{~h}$ post-transfection, resuspended in $50 \mu \mathrm{L}$ PBS, mixed 1:1 (v/v) with $50 \mu \mathrm{L} 0.4 \%(\mathrm{v} / \mathrm{v})$ Trypan Blue Stain (Invitrogen) and incubated for $1 \mathrm{~min}$. Total cell number and number of stained cells of each sample were counted in a hemocytometer. About 100-200 total cells per sample were counted. To obtain the fraction of dead cells, the number of stained cells was divided by the total cell number. For cell viability (MTS) assays, one tenth of the growth medium volume of MTS reagent (CellTiter 96 AQueous One Solution Cell Proliferation Assay by Promega) was added to each well $48 \mathrm{~h}$ post-transfection. Cells were incubated for approximately $1 \mathrm{~h}$ before the absorbance at $495 \mathrm{~nm}$ of the supernatants was measured with a NanoDrop ND-1000 Spectrophotometer (Thermo Scientific, Waltham, MA, USA). Fresh growth medium with the addition of one tenth of MTS reagent was used as blank.

\section{Detection of apoptosis by DNA laddering}

Freshly passaged HeLa cells were seeded into 6-well plates $\left(10^{6}\right.$ cells per well in complete growth medium) and cultivated overnight to $\sim 70 \%$ confluence. Cells were transfected with $50 \mathrm{nM}$ of the indicated ASOs using the DreamFect Gold transfection reagent $(\mathrm{OZ}$ Biosciences) according to the manufacturer's recommendations. Medium was replaced with fresh prewarmed complete growth medium $6 \mathrm{~h}$ post-transfection. At $48 \mathrm{~h}$ post-transfection, cells were lysed and DNA was prepared with the Apoptotic DNA Ladder Kit (Roche, Mannheim, Germany) according to the manufacturer's recommendations. Samples were analyzed using a $1 \%$ agarose TAE gel and DNA was visualized with ethidium bromide.

\section{Additional files}

Additional file 1: Figure S1. (a) Neighbor-joining trees of the flanking 1000 bp upstream (left tree) and downstream (right tree) relative to retroviral orientation. Two pairs of flanking sequences that cluster are highlighted by colored boxes, whereby elements nos. 3 and 43 only clustered in their upstream regions. Element numbers according to Table 1 are shown in parentheses. The horizontal bars represent 0.1 substitutions per nucleotide position. (b) Comparison of the genomic loci representing HML-10 elements nos. 3 and 43 (see blue boxes in panel (a)) that integrated into a $L 1 M B 7$ repeat or into a MER4A1 repeat, respectively. Both HML-10 elements therefore likely represent independent integration events. (c) Comparison of the genomic loci representing HML-10 elements nos. 27 and 45 (red boxes in panel (a)) that are both flanked by LTR5_Hs and HERVK-int repeats in the same configuration. The overall sequence identity of the depicted regions is $>88 \%$. Therefore, one of these $\mathrm{HML}-10$ was likely the result of a chromosomal duplication event that copied the other one. Images in panels (b) and (c) were obtained from the UCSC Genome Browser [68]. (PDF 1217 kb)

Additional file 2: Table S1. Presence or absence of HML-10 in different mammalian genomes. The number of LTR14 hits that represent HML-10 elements in the indicated genomes were assessed with the UCSC Table Browser [67] by querying LTR14 in the respective RepeatMasker tracks [69, 70]. BLAT [74] searches within the UCSC Genome Browser [68] using the consensus sequence of LTR14 obtained from the DFAM database (www.dfam.org) [75] were performed verify the presence of HML-10. (PDF $262 \mathrm{~kb}$ )

Additional file 3: Figure S2. Identification of a putative TSS in the HML-10(DAP3) 5'LTR. The sequence shown is chr1:153935293-153936036 of the human genome hg18 assembly [1]. The HML-10(DAP3) 5'LTR (according to RepeatMasker annotation [70]) is highlighted in bold letters. Six Inr sequences highlighted blue were identified by sequence homology searches of the consensus YYANWYY sequence [76]. Five TATA boxes highlighted red were identified with the TFBind program [77] on http://tfbind.hgc.jp using a similarity threshold of 0.8. Inr2 and TATA4 are overlapping. Only one $\operatorname{lnr}$ element $(\operatorname{Inr} 1)$ is located in close proximity downstream of a TATA box (TATA3). The putative TSS within Inr1 is underlined. A downstream promoter element (DPE) highlighted violet matching the consensus RGWYVT sequence [49] is located $19 \mathrm{bp}$ downstream of the putative TSS. Primer locations for LTRfor1, LTRfor2 and LTRrev (see Additional file 4) are indicated by arrows. An IFNY activated sequence (GAS) is highlighted green. (PDF 364 kb)

Additional file 4: Protein fasta sequences of Pol proteins of different endogenous and exogenous betaretroviruses. (TXT 11 kb)

Additional file 5: Primer sequences. (XLS $42 \mathrm{~kb}$ )

Additional file 6: Sequences of ASOs. Asterisks denote phosphorothioate bonds. (XLS $39 \mathrm{~kb}$ )

\section{Abbreviations}

5'RACE-PCR: 5' rapid amplification of cDNA ends-PCR; ASO: Antisense oligonucleotide; BLAT: BLAST-like Alignment Tool; DAP3: Death-associated protein 3; DMEM: Dulbecco's Modified Eagle's Medium; DPE: Downstream promoter element; DR: Death Receptor; fLuc: Firefly luciferase; GAS: IFNY activated sequence; HERV: Human endogenous retrovirus; HML: Human MMTV-like; HSV-TK: Herpes simplex virus thymidine kinase; HUVEC: Human umbilical vein endothelial cells; IFNy: Interferon-gamma; Inr: Initiator element; JSRV: Jaagsiekte sheep retrovirus; LTR: long terminal repeat; MMTV: Mouse mammary tumor virus; MPMV: Mason-Pfizer monkey virus; Mya: Million years ago; NWM: New World monkey; OWM: Old World monkey; qRT-PCR: Quantitative real-time PCR; RE: Retroelement; rLuc: Renilla luciferase; RT: Reverse transcriptase; TE: Transposable element; TNFa: Tumor necrosis factor-alpha; TSD: Target site duplication; TSS: Transcription start site

\section{Acknowledgements}

We thank Dr. Anja Twiehaus for support in some of the experiments. Prof. Peter $\mathrm{H}$. Seeberger of the Max Planck Institute of Colloids and Interfaces, Potsdam, Germany, is thanked for his generous support of FB.

\section{Funding}

This work was supported by the Max Planck Society and a grant from the Volkswagenstiftung (Lichtenberg Program) to MRS. KM provided some financial support.

\section{Availability of data and materials}

All DNA sequence data is publicly available and was retrieved from the UCSC Genome Browser on https://genome.ucsc.edu/ with the coordinates shown in Table 1. All other data generated or analyzed during this study are included in this published article and its additional files.

\section{Author's contributions}

KM initiated the project. KM, JH and FB designed the experiments. FB performed the experiments and prepared the data with assistance from $\mathrm{KM}, \mathrm{JH}$, $\mathrm{RH}, \mathrm{AF}$ and MRS. HL enabled us to perform these studies. FB and KM wrote the manuscript. All authors read and approved the final version of the manuscript. 


\section{Competing interests}

The authors declare that they have no competing interests.

\section{Consent for publication}

Not applicable.

\section{Ethics approval and consent to participate}

Not applicable.

\section{Author details}

'Max Planck Institute for molecular Genetics, Ihnestr. 63-73, 14195 Berlin, Germany. ${ }^{2}$ Institute of Medical Microbiology, University of Zurich, Gloriastr. 32, 8006 Zurich, Switzerland. ${ }^{3}$ Current affiliation: Max Planck Institute of Colloids and Interfaces, Am Mühlenberg 1, 14424 Potsdam, Germany. ${ }^{4}$ Current affiliation: University of Zurich, Institute of Molecular Life Sciences, Winterthurerstr. 190, 8057 Zurich, Switzerland. ${ }^{5}$ Current affiliation: Functional Epigenomics, CCG, Cologne University Hospital, University of Cologne, Weyertal 115b, 50931 Cologne, Germany. ${ }^{6}$ Dahlem Centre for Genome Research and Medical Systems Biology, Fabeckstr. 60-62, 14195 Berlin, Germany.

\section{Received: 26 July 2016 Accepted: 19 November 2016}

Published online: 07 December 2016

\section{References}

1. Lander ES, Linton LM, Birren B, Nusbaum C, Zody MC, Baldwin J, et al. Initial sequencing and analysis of the human genome. Nature. 2001;409:860-921.

2. de Koning AP, Gu W, Castoe TA, Batzer MA, Pollock DD. Repetitive elements may comprise over two-thirds of the human genome. PLoS Genet. 2011;7:e1002384

3. Gogvadze E, Buzdin A. Retroelements and their impact on genome evolution and functioning. Cell Mol Life Sci. 2009;66:3727-42.

4. Bannert N, Kurth R. The evolutionary dynamics of human endogenous retroviral families. Annu Rev Genomics Hum Genet. 2006;7:149-73.

5. Faulkner GJ, Kimura Y, Daub CO, Wani S, Plessy C, Irvine KM, et al. The regulated retrotransposon transcriptome of mammalian cells. Nat Genet. 2009;41:563-71.

6. Conley AB, Miller WJ, Jordan IK. Human cis natural antisense transcripts initiated by transposable elements. Trends Genet. 2008;24:53-6.

7. Gosenca D, Gabriel U, Steidler A, Mayer J, Diem O, Erben P, et al. HERV-Emediated modulation of PLA2G4A transcription in urothelial carcinoma. PLoS One. 2012;7:e49341.

8. Li F, Nellåker C, Yolken RH, Karlsson H. A systematic evaluation of expression of HERV-W elements; influence of genomic context, viral structure and orientation. BMC Genomics. 2011:12:22.

9. Schön U, Diem O, Leitner L, Günzburg WH, Mager DL, Salmons B, et al. Human endogenous retroviral long terminal repeat sequences as cell typespecific promoters in retroviral vectors. J Virol. 2009;83:12643-50.

10. Huh JW, Kim DS, Kang DW, Ha HS, Ahn K, Noh YN, et al. Transcriptional regulation of GSDML gene by antisense-oriented HERV-H LTR element. Arch Virol. 2008;153:1201-5.

11. Sin HS, Huh JW, Kim DS, Kang DW, Min DS, Kim TH, et al. Transcriptional control of the HERV-H LTR element of the GSDML gene in human tissues and cancer cells. Arch Virol. 2006:151:1985-94.

12. Sjøttem E, Anderssen S, Johansen T. The promoter activity of long terminal repeats of the HERV-H family of human retrovirus-like elements is critically dependent on Sp1 family proteins interacting with a GC/GT box located immediately 3' to the TATA box. J Virol. 1996;70:188-98.

13. Chang NT, Yang WK, Huang HC, Yeh KW, Wu CW. The transcriptional activity of HERV-I LTR is negatively regulated by its cis-elements and wild type p53 tumor suppressor protein. J Biomed Sci. 2007;14:211-22.

14. Gogvadze E, Stukacheva E, Buzdin A, Sverdlov E. Human-specific modulation of transcriptional activity provided by endogenous retroviral insertions. J Virol. 2009;83:6098-105.

15. Buzdin A, Kovalskaya-Alexandrova E, Gogvadze E, Sverdlov E. At least $50 \%$ of human-specific HERV-K (HML-2) long terminal repeats serve in vivo as active promoters for host nonrepetitive DNA transcription. J Virol. 2006:80:10752-62.

16. Ruda VM, Akopov SB, Trubetskoy DO, Manuylov NL, Vetchinova AS, Zavalova $L L$, et al. Tissue specificity of enhancer and promoter activities of a HERV-K(HML-2) LTR. Virus Res. 2004;104:11-6.

17. Domansky AN, Kopantzev EP, Snezhkov EV, Lebedev YB, Leib-Mosch C, Sverdlov ED. Solitary HERV-K LTRs possess bi-directional promoter activity and contain a negative regulatory element in the $\mathrm{U} 5$ region. FEBS Lett. 2000:42:191-5.

18. Dangel AW, Mendoza AR, Baker BJ, Daniel CM, Carroll MC, Wu LC, et al. The dichotomous size variation of human complement $C 4$ genes is mediated by a novel family of endogenous retroviruses, which also establishes speciesspecific genomic patterns among Old World primates. Immunogenetics. 1994;40:425-36.

19. Mack M, Bender K, Schneider PM. Detection of retroviral antisense transcripts and promoter activity of the HERV-K(C4) insertion in the MHC class III region. Immunogenetics. 2004;56:321-32.

20. Kissil JL, Deiss LP, Bayewitch M, Raveh T, Khaspekov G, Kimchi A. Isolation of DAP3, a novel mediator of interferon-gamma-induced cell death. J Biol Chem. 1995:270:27932-6.

21. Miyazaki T, Reed JC. A GTP-binding adapter protein couples TRAIL receptors to apoptosis-inducing proteins. Nat Immunol. 2001;2:493-500.

22. Seifarth W, Frank O, Zeilfelder U, Spiess B, Greenwood AD, Hehlmann R, et al. Comprehensive analysis of human endogenous retrovirus transcriptional activity in human tissues with a retrovirus-specific microarray. J Virol. 2005;79:341-52.

23. Seifarth W, Spiess B, Zeilfelder U, Speth C, Hehlmann R, Leib-Mösch C. Assessment of retroviral activity using a universal retrovirus chip. J Virol Methods. 2003;112:79-91.

24. Frank O, Giehl M, Zheng C, Hehlmann R, Leib-Mösch C, Seifarth W. Human endogenous retrovirus expression profiles in samples from brains of patients with schizophrenia and bipolar disorders. J Virol. 2005;79:10890-901.

25. Frank O, Verbeke C, Schwarz N, Mayer J, Fabarius A, Hehlmann R, et al. Variable transcriptional activity of endogenous retroviruses in human breast cancer. J Virol. 2008:82:1808-18.

26. Schön U, Seifarth W, Baust C, Hohenadl C, Erfle V, Leib-Mösch C. Cell typespecific expression and promoter activity of human endogenous retroviral long terminal repeats. Virology. 2001;279:280-91

27. Haupt S, Tisdale M, Vincendeau M, Clements MA, Gauthier DT, Lance R, et al. Human endogenous retrovirus transcription profiles of the kidney and kidney-derived cell lines. J Gen Virol. 2011:92:2356-66.

28. Johnson WE, Coffin JM. Constructing primate phylogenies from ancient retrovirus sequences. Proc Natl Acad Sci U S A. 1999:96:10254-60.

29. Mayer J, Meese EU. The human endogenous retrovirus family HERV-K(HML3). Genomics. 2002:80:331-43.

30. Seifarth W, Baust C, Murr A, Skladny H, Krieg-Schneider F, Blusch J, et al. Proviral structure, chromosomal location, and expression of HERV-K-T47D, a novel human endogenous retrovirus derived from T47D particles. J Virol. 1998;72:8384-91.

31. Medstrand P, Mager DL, Yin H, Dietrich U, Blomberg J. Structure and genomic organization of a novel human endogenous retrovirus family: HERV-K (HML-6). J Gen Virol. 1997:78:1731-44.

32. Lavie L, Medstrand P, Schempp W, Meese E, Mayer J. Human endogenous retrovirus family HERV-K(HML-5): status, evolution, and reconstruction of an ancient betaretrovirus in the human genome. J Virol. 2004;78:8788-98.

33. Pruitt KD, Tatusova T, Maglott DR. NCBI Reference Sequence (RefSeq): a curated non-redundant sequence database of genomes, transcripts and proteins. Nucleic Acids Res. 2005;33(Database issue):D501-4.

34. Brady T, Lee YN, Ronen K, Malani N, Berry CC, Bieniasz PD, et al. Integration target site selection by a resurrected human endogenous retrovirus. Genes Dev. 2009;23:633-43.

35. Bushman FD. Targeting survival. Integration site selection by retroviruses and LTR-retrotransposons. Cell. 2003;115:135-8.

36. Smit AF. Interspersed repeats and other momentos of transposable elements in mammalian genomes. Curr Opin Genet Dev. 1999;9:657-63.

37. Medstrand $\mathrm{P}$, van de Lagemaat LN, Mager DL. Retroelement distrubutions in the human genome: Variations associate with age and proximity to genes. Genome Res. 2002;12:1483-95.

38. Villesen $\mathrm{P}$, Aagaard L, Wiuf C, Pedersen FS. Identification of endogenous retroviral reading frames in the human genome. Retrovirology. 2004;1:32.

39. Conley $A B$, Jordan IK. Cell type-specific termination of transcription by transposable element sequences. Mob DNA. 2012;3:15

40. Assinger A, Yaiw KC, Göttesdorfer I, Leib-Mösch C, Söderberg-Nauclér C. Human cytomegalovirus (HCMV) induces human endogenous retrovirus (HERV) transcription. Retrovirology. 2013;10:132.

41. Maliniemi P, Vincendeau M, Mayer J, Frank O, Hahtola S, Karenko L, et al. Expression of human endogenous retrovirus-w including syncytin-1 in cutaneous T-cell lymphoma. PLoS One. 2013;8:e76281. 
42. Diem O, Schäffner M, Seifarth W, Leib-Mösch C. Influence of antipsychotic drugs on human endogenous retrovirus (HERV) transcription in brain cells PLoS One. 2012;7:e30054

43. Frank O, Jones-Brando L, Leib-Mosch C, Yolken R, Seifarth W. Altered transcriptional activity of human endogenous retroviruses in neuroepithelial cells after infection with Toxoplasma gondii. J Infect Dis. 2006;194:1447-9.

44. Dunn CA, Romanish MT, Gutierrez LE, van de Lagemaat LN, Mager DL. Transcription of two human genes from a bidirectional endogenous retrovirus promoter. Gene. 2006;366:335-42.

45. Boehm U, Klamp T, Groot M, Howard JC. Cellular responses to interferongamma. Annu Rev Immunol. 1997;15:749-95.

46. Harms JS, Splitter GA. Interferon-gamma inhibits transgene expression driven by SV40 or CMV promoters but augments expression driven by the mammalian MHC I promoter. Hum Gene Ther. 1995;6:1291-7.

47. Kulics J, Colten HR, Perlmutter DH. Counterregulatory effects of interferon-gamma and endotoxin on expression of the human C4 genes. J Clin Invest. 1990;85:943-9.

48. Köhrer K, Grummt I, Horak I. Functional RNA polymerase II promoters in solitary retroviral long terminal repeats (LTR-IS elements). Nucleic Acids Res. 1985;13:2631-45.

49. Butler JE, Kadonaga JT. The RNA polymerase II core promoter: a key component in the regulation of gene expression. Genes Dev. 2002;16:2583-92

50. Kowalskaya E, Buzdin A, Gogvadze E, Vinogradova T, Sverdlov E. Functional human endogenous retroviral LTR transcription start sites are located between the $\mathrm{R}$ und $\mathrm{U} 5$ regions. Virology. 2006;346:373-8.

51. Zong X, Huang L, Tripathi V, Peralta R, Freier SM, Guo S, et al. Knockdown of nuclear-retained long noncoding RNAs using modified DNA antisense oligonucleotides. Methods Mol Biol. 2015;1262:321-31.

52. Gagnon KT, Li L, Chu Y, Janowski BA, Corey DR. RNAi factors are present and active in human cell nuclei. Cell Rep. 2014;6:211-21.

53. Kwok T, Heinrich J, Jung-Shiu J, Meier MG, Mathus S, Moelling K. Reduction of gene expression by a hairpin-loop structured oligodeoxynucleotide: alternative to siRNA and antisense. Biochim Biophys Acta. 2009;1790:1170-8.

54. Faghihi MA, Wahlestedt C. Regulatory roles of natural antisense transcripts. Nat Rev Mol Cell Biol. 2009;10:637-43.

55. Modarresi F, Faghihi MA, Lopez-Toledano MA, Fatemi RP, Magistri M, Brothers SP, van der Brug MP, Wahlestedt C. Inhibition of natural antisense transcripts in vivo results in gene-specific transcriptional upregulation. Nat Biotechnol. 2012;30:453-9.

56. Brink AA, Oudejans JJ, Jiwa M, Walboomers JM, Meijer CJ, van den Brule AJ. Multiprimed cDNA synthesis followed by PCR is the most suitable method for Epstein-Barr virus transcript analysis in small lymphoma biopsies. Mol Cell Probes. 1997;11:39-47.

57. Hanahan D, Weinberg RA. Hallmarks of cancer: the next generation. Cell. 2011;144:646-74.

58. Jia Y, Ye L, Ji K, Zhang L, Hargest R, Ji J, et al. Death associated protein-3, DAP-3, correlates with preoperative chemotherapy effectiveness and prognosis of gastric cancer patients following perioperative chemotherapy and radical gastrectomy. Br J Cancer. 2014;110:421-9.

59. Wazir U, Jiang WG, Sharma AK, Mokbel K. The mRNA expression of DAP3 in breast cancer: correlation with clinicopathological parameters. Anticancer Res. 2012;32:671-4.

60. Sasaki H, Ide N, Yukiue H, Kobayashi Y, Fukai I, Yamakawa Y, et al. Arg and DAP3 expression was correlated with human thymoma stage. Clin Exp Metastasis. 2004;21:507-13.

61. Jacques C, Fontaine JF, Franc B, Mirebeau-Prunier D, Triau S, Savagner F, et al. Death-associated protein 3 is overexpressed in human thyroid oncocytic tumours. Br J Cancer. 2009;101:132-8.

62. Davidsson J, Andersson A, Paulsson K, Heidenblad M, Isaksson M, Borg A, et al. Tiling resolution array comparative genomic hybridization, expression and methylation analyses of dup(1q) in Burkitt lymphomas and pediatric high hyperdiploid acute lymphoblastic leukemias reveal clustered nearcentromeric breakpoints and overexpression of genes in 1q22-32.3. Hum Mol Genet. 2007;16:2215-25.

63. Mariani L, Beaudry C, McDonough WS, Hoelzinger DB, Kaczmarek E, Ponce $F$, et al. Death-associated protein 3 (Dap-3) is overexpressed in invasive glioblastoma cells in vivo and in glioma cell lines with induced motility phenotype in vitro. Clin Cancer Res. 2001;7:2480-9.

64. Wilson AS, Power BE, Molloy PL. DNA hypomethylation and human diseases. Biochim Biophys Acta. 2007;1775:138-62.

65. Yoder JA, Walsh CP, Bestor TH. Cytosine methylation and the ecology of intragenomic parasites. Trends Genet. 1997;8:335-40.
66. Wilkins AS. The enemy within: an epigenetic role of retrotransposon in cancer initiation. Bioessays. 2010;32:856-65.

67. Karolchik D, Hinrichs AS, Furey TS, Roskin KM, Sugnet CW, Haussler D, et al. The UCSC Table Browser data retrieval tool. Nucleic Acids Res. 2004;32:D493-6.

68. Kent WJ, Sugnet CW, Furey TS, Roskin KM, Pringle TH, Zahler AM, et al. The human genome browser at UCSC. Genome Res. 2002;12:996-1006.

69. Jurka J, Kapitonov W, Pavlicek A, Klonowski P, Kohany O, Walichiewicz J. Repbase Update, a database of eukaryotic repetitive elements. Cytogenet Genome Res. 2005;110:462-7.

70. Smit AFA, Hublez R, Green P. RepeatMasker Open-3.0. 1996-2010<www. repeatmasker.org>.

71. Larkin MA, Blackshields G, Brown NP, Chenna R, McGettigan PA, McWilliam $H$, et al. Clustal W and Clustal X version 2.0. Bioinformatics. 2007;23:2947-8.

72. Jern P, Sperber GO, Blomberg J. Use of endogenous retroviral sequences (ERVs) and structural markers for retroviral phylogenetic inference and taxonomy. Retrovirology. 2005;2:50.

73. TreeView 1.6.6 Software. http://taxonomy.zoology.gla.ac.uk/rod/treeview. html. Accessed Oct 2015

74. Kent WJ. BLAT-the BLAST-like alignment tool. Genome Res. 2002;12:656-64.

75. Hubley R, Finn RD, Clements J, Eddy SR, Jones TA, Bao W, Smit AF, Wheeler TJ. The Dfam database of repetitive DNA families. Nucleic Acids Res. 2016; 44(D1):D81-9.

76. Xi H, Yu Y, Fu Y, Foley J, Halees A, Weng Z. Analysis of overrepresented motifs in human core promoters reveals dual regulatory roles of $Y Y 1$. Genome Res. 2007;17:798-806.

77. Tsunoda T, Takagi T. Estimating transcription factor bindability on DNA. Bioinformatics. 1999;15:622-30.

78. Steiper ME, Young ME. Primate molecular divergence dates. Mol Phylogenet Evol. 2006;41:384-94

79. Seifarth W, Krause U, Hohenadl C, Baust C, Hehlmann R, Leib-Mösch C. Rapid identification of all known retroviral reverse transcriptase sequences with a novel versatile detection assay. AIDS. 2000;16:721-9.

\section{Submit your next manuscript to BioMed Central and we will help you at every step:}

- We accept pre-submission inquiries

- Our selector tool helps you to find the most relevant journal

- We provide round the clock customer support

- Convenient online submission

- Thorough peer review

- Inclusion in PubMed and all major indexing services

- Maximum visibility for your research

Submit your manuscript at www.biomedcentral.com/submit
C BioMed Central 